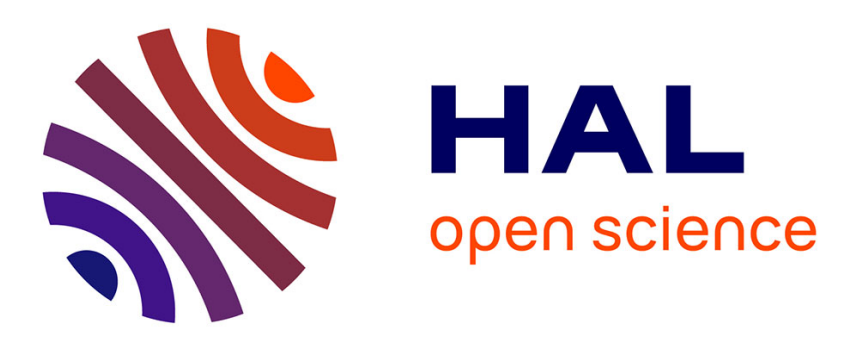

\title{
Homogenization of a micropolar fluid past a porous media with non-zero spin boundary condition
}

\author{
Francisco J. Suárez-Grau
}

\section{To cite this version:}

Francisco J. Suárez-Grau. Homogenization of a micropolar fluid past a porous media with non-zero spin boundary condition. 2020. hal-02918354

\section{HAL Id: hal-02918354 \\ https://hal.science/hal-02918354}

Preprint submitted on 20 Aug 2020

HAL is a multi-disciplinary open access archive for the deposit and dissemination of scientific research documents, whether they are published or not. The documents may come from teaching and research institutions in France or abroad, or from public or private research centers.
L'archive ouverte pluridisciplinaire HAL, est destinée au dépôt et à la diffusion de documents scientifiques de niveau recherche, publiés ou non, émanant des établissements d'enseignement et de recherche français ou étrangers, des laboratoires publics ou privés. 


\title{
Homogenization of a micropolar fluid past a porous media with non-zero spin boundary condition
}

\author{
Francisco Javier SUÁREZ-GRAU*
}

\begin{abstract}
We consider a micropolar fluid flow in a media perforated by periodically distributed obstacles of size $\varepsilon$. A non-homogeneous boundary condition for microrotation is considered: the microrotation is assumed to be proportional to the rotation rate of the velocity on the boundary of the obstacles. The existence and uniqueness of solution is analyzed. Moreover, passing to the limit when $\varepsilon$ tends to zero, an analogue of the classical micropolar Darcy law in the theory of porous media is derived.
\end{abstract}

AMS classification numbers: 76A05, 76M50, 76S05, 35B27, 35Q35.

Keywords: Homogenization; micropolar fluid; Darcy's law; porous media; non-zero spin boundary condition.

\section{Introduction}

Micropolar fluid model is a non-Newtonian model which represents a generalization of the well-established Newtonian Navier-Stokes model which takes into account the microstructure of the fluid. It describes the behavior of numerous real fluids (e.g. polymeric suspensions, liquid crystals, muddy fluids, animal blood, etc.) better than the classical one. The related mathematical model expresses the balance of momentum, mass and angular momentum. Thus, a new unknown function $\hat{w}$ called microrotation (i.e. the angular velocity field of rotation of particles) is added to the classical pressure $\hat{p}$ and velocity $\hat{u}$. Consequently, Newtonian Navier-Stokes equations become coupled with a new vector equation coming from the conservation of angular momentum, see Eringen [25] and Lukaszewicz [29]. In view of its application in porous media, we can assume a small Reynolds number and neglect the nonlinear terms and so the following micropolar equations are considered

$$
\begin{array}{r}
-\left(\nu+\nu_{r}\right) \Delta \hat{u}+\nabla \hat{p}=2 \nu_{r} \operatorname{rot}(\hat{w})+\hat{f}, \\
\operatorname{div}(\hat{u})=0, \\
-\left(c_{a}+c_{d}\right) \Delta \hat{w}+4 \nu_{r} \hat{w}=2 \nu_{r} \operatorname{rot}(\hat{u})+\hat{g} .
\end{array}
$$

The different viscosities $\nu, \nu_{r}, c_{a}$ and $c_{d}$ are strictly positive and characterize the isotropic properties of the fluid, $f$ represents the body force and $g$ the body torque.

Solution to the governing equations in the presence of solid boundaries requires imposing appropriate boundary conditions. Typical conditions on the solid boundary are the no-slip condition for velocity and the no-spin condition for microrotation, which read

$$
\hat{u}=0 \text { and } \hat{w}=0
$$

which respectively imply that the fluid adheres to the solid boundary and that the fluid elements can not rotate on the fluid-solid interface. However, a more general boundary condition for microrotation was introduced to take into account the rotation of the microelements on the solid boundary, which is effectively proved to be in good

\footnotetext{
*Departamento de Ecuaciones Diferenciales y Análisis Numérico. Facultad de Matemáticas. Universidad de Sevilla. 41012-Sevilla (Spain) fjsgrau@us.es
} 
accordance with experiments, see Bessonov [11, 12] and Migun et al. [30, 31]. This condition, called non-zero spin condition, reads

$$
\hat{w} \times n=\frac{\alpha}{2} \operatorname{rot}(\hat{u}) \times n, \quad \hat{w} \cdot n=0,
$$

where $n$ is a normal unit vector to the boundary and the coefficient $\alpha$ describes the interaction between the given fluid and solid.

It should be noted that in the previous studies the no-slip condition for velocity on the solid surface combined with non-zero spin condition (1.1) for microrotation is assumed. However slippage is experimentally observed in various systems at fluid-solid interfaces and can strongly influence hydrodynamic behavior in microscale and nanoscale flows. For this reason, no-slip condition for velocity should be replaced by a more general relation. In this sense, several boundary conditions have been considered to model the observed slippage, most of them include limited yield stress or retain slippage value proportional to the shear stress. But there is a new interpretation of the observed slippage in micropolar fluids, expressed in terms of the microrotation, by introducing a new slippage condition for velocity compatible with non-zero spin boundary condition for microrotation. This condition was proposed in Bayada et al. [6, 7] in the framework of lubrication, and allows a slippage in the tangential direction and retains a non-penetration condition in the normal direction $n$ ( $\delta$ is a real parameter)

$$
\hat{u} \times n=\delta \operatorname{rot}(\hat{w}) \times n, \quad \hat{u} \cdot n=0 .
$$

On the other hand, the behavior of fluid flows in porous media is of great importance in industrial and engineering applications. As is well known, classical (Newtonian) Darcy's law is generally considered for modelling of flow through a porous media, see Darcy [23]. By using homogenization techniques, the mathematical derivation of such Darcy's law was obtained in Tartar [32] assuming no-slip boundary conditions

$$
\hat{u}=K(\hat{f}-\nabla \hat{p}), \quad \operatorname{div}(\hat{u})=0,
$$

where the matrix coefficient $K$ is calculated by using Newtonian local problems. In addition, problems with different types of slippage conditions for Newtonian fluids in porous media have been studied by several authors giving rise to a wide range of Darcy's laws. More precisely, the Navier-Stokes (or Stokes) flow in a periodic porous media with Fourier boundary conditions on the boundary of the obstacles was studied in Conca [21] by using the method of oscillating test functions and two-scale method with asymptotic expansion of the solution. The case of classical slip boundary conditions was treated in Allaire [2] by means of the method of oscillating test functions. Finally, the case of non-homogeneous slip boundary conditions was considered in Cioranescu et al. [16] combining the method of oscillating test functions with the technique introduced in Vanninathan [34] to treat the surface integrals. Finally, the case of non-homogeneous slip boundary conditions was revisited in Capatina and Ene [14] and Zaki [35] by using the periodic unfolding method together with the boundary unfolding operator which allows to treat quite elementary the surface integrals, see Cioranescu et al. [15, 17, 19].

Although the behavior of micropolar fluid flows in porous media become of great practical relevance, the literature on the modelling of such type of problem by homogenization methods is far less complete. Lukaszewicz [29] rigorously derived the following version of the classical Darcy law by using the two-scale convergence method

$$
\hat{u}=K^{(1)}(\hat{f}-\nabla \hat{p})+K^{(2)} \hat{g}, \quad \operatorname{div}(\hat{u})=0, \quad \hat{w}=L^{(1)}(\hat{f}-\nabla \hat{p})+L^{(2)} \hat{g},
$$

where the matrix coefficients $K^{(k)}$ and $L^{(k)}, k=1,2$, are calculated by using micropolar local problems. We also refer to Aganovic and Tutek [3] for the nonstationary case and to Bayada et al. [9] for the micropolar effects in the coupling of a thin film past a porous media.

Previous studies obtained different Darcy's laws for micropolar fluids by assuming on the obstacles of the porous media the no-slip conditon for velocity and the no-spin condition for microrotation, not allowing to capture the microscopic behavior of the fluid near the boundary of the obstacles. Thus, the goal of this paper is first to establish existence and uniqueness of solution of the micropolar system in the considered porous media by assuming non-zero spin boundary condition (1.1) and new slippage condition (1.2) on the boundary of the obstacles, and then to derive a generalized Darcy's law by means of a combination of the periodic unfolding method with the boundary unfolding 
operator to treat the surface integrals. As far as the author knows, this is the first attempt to carry out such an homogenization analysis for micropolar fluids in porous media, which is the main novelty of the work, and could be instrumental for understanding the effects on this type of non-Newtonian fluid flows taking into account the boundary of the obstacles.

The structure of the paper is as follows. In Section 2, we make an introduction of the problem and its setting. In Section 3, we give the main results of the paper, i.e. the existence and uniqueness of solution (Theorem 3.2) and the asymptotic behavior of the solution (Theorem 3.3). The proof of the corresponding results are given in Section 4. The paper ends with a list of references.

\section{The setting of the problem}

Definition of the domain. Let $\Omega$ be a bounded connected open set in $\mathbb{R}^{3}$, with smooth enough boundary $\partial \Omega$. Denote $Y=(0,1)^{3}$ and $F$ an open connected subset of $Y$ with a $C^{1,1}$ boundary $\partial F$, such that $\bar{F} \subset Y$. We denote $Y^{*}=Y \backslash \bar{F}$.

For $k \in \mathbb{Z}^{2}$, each cell $Y_{k, \varepsilon}=\varepsilon k+\varepsilon Y$ is similar to the unit cell $Y$ rescaled to size $\varepsilon$ and $F_{k, \varepsilon}=\varepsilon k+\varepsilon F$ is similar to $F$ rescaled to size $\varepsilon$. We denote $Y_{k, \varepsilon}^{*}=Y_{k, \varepsilon} \backslash \bar{F}_{k, \varepsilon}$.

We denote by $\tau\left(\bar{F}_{k, \varepsilon}\right)$ the set of all translated images of $\bar{F}_{k, \varepsilon}$. The set $\tau\left(\bar{F}_{k, \varepsilon}\right)$ represents the obstacles in $\mathbb{R}^{3}$. The porous media is defined by $\Omega_{\varepsilon}=\Omega \backslash \bigcup_{k \in \mathcal{K}_{\varepsilon}} \bar{F}_{k, \varepsilon}$, where $\mathcal{K}_{\varepsilon}:=\left\{k \in \mathbb{Z}^{N}: Y_{k, \varepsilon} \cap \Omega \neq \emptyset\right\}$. By this construction, $\Omega_{\varepsilon}$ is a periodically perforated domain with obstacles of the same size as the period.

We make the assumption that the obstacles $\tau\left(\bar{F}_{k, \varepsilon}\right)$ do no intersect the boundary $\partial \Omega$. We denote by $F_{\varepsilon}$ the set of all the obstacles contained in $\Omega_{\varepsilon}$. Then, $F_{\varepsilon}=\cup_{k \in \mathcal{K}_{\varepsilon}} \bar{F}_{k, \varepsilon}$.

We define $n$ the outside normal vector to $\partial F$. We denote by $n_{\varepsilon}(x)=n(x / \varepsilon)$ the outside normal vector (extended by periodicity) to $\partial F_{\varepsilon}$.

Statement of the problem. We consider that the micropolar fluid flow is described by the following linearized micropolar equations in $\Omega_{\varepsilon}$, taking into account the dependence of $\varepsilon$,

$$
\begin{array}{rc}
-\left(\nu+\nu_{r}\right) \Delta \hat{u}_{\varepsilon}+\nabla \hat{p}_{\varepsilon}=2 \nu_{r} \operatorname{rot}\left(\hat{w}_{\varepsilon}\right)+\hat{f}_{\varepsilon} & \text { in } \quad \Omega_{\varepsilon}, \\
\operatorname{div}\left(\hat{u}_{\varepsilon}\right)=0 & \text { in } \Omega_{\varepsilon}, \\
-\left(c_{a}+c_{d}\right) \Delta \hat{w}_{\varepsilon}+4 \nu_{r} \hat{w}_{\varepsilon}=2 \nu_{r} \operatorname{rot}\left(\hat{u}_{\varepsilon}\right)+\hat{g}_{\varepsilon} & \text { in } \quad \Omega_{\varepsilon} .
\end{array}
$$

As discussed in the introduction, we impose the following boundary conditions for velocity and microrotation on the surface of the obstacles

$$
\begin{array}{rr}
\hat{w}_{\varepsilon} \times n_{\varepsilon}=\frac{\alpha}{2} \operatorname{rot}\left(\hat{u}_{\varepsilon}\right) \times n_{\varepsilon} & \text { on } \partial F_{\varepsilon}, \\
\operatorname{rot}\left(\hat{w}_{\varepsilon}\right) \times n_{\varepsilon}=\frac{2 \nu_{r}}{c_{a}+c_{d}} \beta\left(\hat{u}_{\varepsilon} \times n_{\varepsilon}\right) & \text { on } \partial F_{\varepsilon}, \\
\hat{u}_{\varepsilon} \cdot n_{\varepsilon}=0 & \text { on } \partial F_{\varepsilon}, \\
\hat{w}_{\varepsilon} \cdot n_{\varepsilon}=0 & \text { on } \partial F_{\varepsilon},
\end{array}
$$

and the homogeneous boundary conditions on the exterior boundary

$$
\hat{u}_{\varepsilon}=0, \quad \hat{w}_{\varepsilon}=0 \quad \text { on } \partial \Omega .
$$

Notice that the usual no-slip and no-spin boundary conditions for the velocity and microrotation are prescribed in the exterior boundary, while non-zero spin and new slip boundary conditions are imposed on the boundary of the 
obstacles. The coefficient $\alpha>0$ appearing in (2.6) describes the interaction between the given fluid and solid, it characterizes microrotation retardation on the solid surfaces. In [11] it was proposed to connect it with the different viscosity coefficients, which allows to give a certain physical sense and to determine the real limits of its value. The coefficient $\beta>0$ in (2.7) is a characteristic of a slippage and allows the control of the slippage at the boundary of the obstacles when the value $\hat{u}_{\varepsilon}$ is not zero.

Mathematical justification of the new slip boundary condition (2.7). By assuming condition (2.6), the supplementary condition (2.7) on the boundary of the obstacles is needed to close the system. The derivation of such boundary condition follows arguments from [6] which is given in the context of lubrication by applying the non-zero spin condition to a flat surface. The idea is to consider $\psi \in H^{1}\left(\Omega_{\varepsilon}\right)^{3}, \psi=0$ on $\partial \Omega$ and $\psi \cdot n_{\varepsilon}=0$ on $\partial F_{\varepsilon}$ and recall the following identities

$$
-\Delta \varphi=\operatorname{rot}(\operatorname{rot}(\varphi))-\nabla \operatorname{div}(\varphi) \quad \forall \varphi \in \mathcal{D}\left(\Omega_{\varepsilon}\right)^{3},
$$

and

$$
\operatorname{div}(\varphi \times \psi)=\psi \cdot \operatorname{rot}(\varphi)-\varphi \cdot \operatorname{rot}(\psi) .
$$

Integrating by parts and taking into account the divergence theorem, we have

$$
\int_{\Omega_{\varepsilon}} \operatorname{div}(\varphi \times \psi) d x=\int_{\partial \Omega}(\varphi \times \psi) \cdot n_{\varepsilon} d \sigma-\int_{\partial F_{\varepsilon}}(\varphi \times \psi) \cdot n_{\varepsilon} d \sigma=\int_{\partial F_{\varepsilon}}\left(\varphi \times n_{\varepsilon}\right) \cdot \psi d \sigma,
$$

and taking into account the last identity (2.12), we get

$$
\int_{\Omega_{\varepsilon}} \operatorname{rot}(\varphi) \cdot \psi d x=\int_{\Omega_{\varepsilon}} \operatorname{rot}(\psi) \cdot \varphi d x+\int_{\partial F_{\varepsilon}}\left(\varphi \times n_{\varepsilon}\right) \cdot \psi d \sigma \quad \forall(\varphi, \psi) \in H^{1}\left(\Omega_{\varepsilon}\right)^{3} \times H^{1}\left(\Omega_{\varepsilon}\right)^{3} .
$$

Thus, multiplying (2.5) by test function $\psi$ and using identities (2.11) and (2.13), we get

$$
\begin{aligned}
& \left(c_{a}+c_{d}\right)\left(\int_{\Omega_{\varepsilon}} \operatorname{rot}\left(\hat{w}_{\varepsilon}\right) \cdot \operatorname{rot}(\psi) d x+\int_{\Omega_{\varepsilon}} \operatorname{div}\left(\hat{w}_{\varepsilon}\right) \cdot \operatorname{div}(\psi) d x+\int_{\partial F_{\varepsilon}}\left(\operatorname{rot}\left(\hat{w}_{\varepsilon}\right) \times n_{\varepsilon}\right) \cdot \psi d \sigma\right) \\
& +4 \nu_{r} \int_{\Omega_{\varepsilon}} \hat{w}_{\varepsilon} \cdot \psi d x=2 \nu_{r}\left(\int_{\Omega_{\varepsilon}} \operatorname{rot}(\psi) \cdot \hat{u}_{\varepsilon} d x+\int_{\partial F_{\varepsilon}}\left(\hat{u}_{\varepsilon} \times n_{\varepsilon}\right) \cdot \psi d \sigma\right)+\int_{\Omega_{\varepsilon}} \hat{g}_{\varepsilon} \cdot \psi d x
\end{aligned}
$$

In this equation, the unknown terms $\operatorname{rot}\left(\hat{w}_{\varepsilon}\right)$ on $\partial F_{\varepsilon}$ prevent a well-posed variational formulation being obtained. It is then possible to cancel the boundary terms on $\partial F_{\varepsilon}$ by assuming

$$
\operatorname{rot}\left(\hat{w}_{\varepsilon}\right) \times n_{\varepsilon}=\frac{2 \nu_{r}}{c_{a}+c_{d}}\left(\hat{u}_{\varepsilon} \times n_{\varepsilon}\right) .
$$

Finally, similarly to [6], we assume the slippage condition (2.7) on the boundary of the obstacles $\partial F_{\varepsilon}$ by introducing an additional parameter $\beta>0$ which will enable the influence of this new condition to be controlled when the value $\hat{u}_{\varepsilon}$ is not zero.

Dimensionless equations. It has been observed (see e.g. $[8,10])$ that the magnitude of the viscosity coefficients appearing in equations (2.3)-(2.5) may influence the effective flow. Thus, it is reasonable to work with the system written in a non-dimensional form. In view of that, we introduce

$$
u_{\varepsilon}=\hat{u}_{\varepsilon}, \quad p_{\varepsilon}=\frac{\hat{p}_{\varepsilon}}{\nu+\nu_{r}}, \quad w_{\varepsilon}=\hat{w}_{\varepsilon}, \quad f_{\varepsilon}=\frac{\hat{f}_{\varepsilon}}{\nu+\nu_{r}}, \quad g_{\varepsilon}=\frac{\hat{g}_{\varepsilon}}{\nu+\nu_{r}}, \quad N^{2}=\frac{\nu_{r}}{\nu+\nu_{r}}, \quad R_{M}=\frac{c_{a}+c_{d}}{\nu+\nu_{r}} .
$$

Dimensionless (non-Newtonian) parameter $N^{2}$ characterizes the coupling between the equations for the velocity and microrotation and it is of order $\mathcal{O}(1)$, in fact $N^{2}$ lies between zero and one. The second dimensionless parameter, 
denoted by $R_{M}$ is related to the characteristic length of the microrotation effects and is compared with small parameter $\varepsilon$. Thus, we assume that $R_{M}=\mathcal{O}\left(\varepsilon^{2}\right)$, namely

$$
R_{M}=\varepsilon^{2} R_{c}, \quad \text { with } R_{c}=\mathcal{O}(1) .
$$

This case is the situation that is commonly introduced to study the micropolar fuid because the angular momentum equation shows a strong coupling between velocity and microrotation in the limit, see $[3,29]$.

The flow equations (2.3)-(2.5) now have the following form

$$
\begin{array}{rc}
-\Delta u_{\varepsilon}+\nabla p_{\varepsilon}=2 N^{2} \operatorname{rot}\left(w_{\varepsilon}\right)+f_{\varepsilon} & \text { in } \Omega_{\varepsilon}, \\
\operatorname{div}\left(u_{\varepsilon}\right)=0 & \text { in } \Omega_{\varepsilon}, \\
-\varepsilon^{2} R_{c} \Delta w_{\varepsilon}+4 N^{2} w_{\varepsilon}=2 N^{2} \operatorname{rot}\left(u_{\varepsilon}\right)+g_{\varepsilon} & \text { in } \Omega_{\varepsilon} .
\end{array}
$$

Concerning the body force and body torque, in order to obtain appropriate estimates, given $f, g \in L^{2}(\Omega)^{3}$ we make the following assumptions

$$
f_{\varepsilon}(x)=\varepsilon^{-1} f(x), \quad g_{\varepsilon}(x)=g(x), \quad \text { a.e. } x \in \Omega_{\varepsilon} .
$$

The corresponding boundary conditions on the boundary of the obstacles read

$$
\begin{array}{rr}
w_{\varepsilon} \times n_{\varepsilon}=\frac{\alpha}{2} \operatorname{rot}\left(u_{\varepsilon}\right) \times n_{\varepsilon} & \text { on } \partial F_{\varepsilon}, \\
\operatorname{rot}\left(w_{\varepsilon}\right) \times n_{\varepsilon}=\frac{2 N^{2}}{\varepsilon^{2} R_{c}} \beta\left(u_{\varepsilon} \times n_{\varepsilon}\right) & \text { on } \partial F_{\varepsilon}, \\
u_{\varepsilon} \cdot n_{\varepsilon}=0 & \text { on } \partial F_{\varepsilon}, \\
w_{\varepsilon} \cdot n_{\varepsilon}=0 & \text { on } \partial F_{\varepsilon},
\end{array}
$$

and the boundary conditions on the exterior boundary read as follows

$$
u_{\varepsilon}=0, \quad w_{\varepsilon}=0 \quad \text { on } \partial \Omega .
$$

Functional setting. Due to the boundary conditions (2.21) and (2.22), we introduce the functional spaces $V_{\varepsilon}$ and $V_{\varepsilon}^{0}$ given by

$$
V_{\varepsilon}=\left\{\varphi \in H^{1}\left(\Omega_{\varepsilon}\right)^{3}: \varphi=0 \text { on } \partial \Omega, \quad \varphi \cdot n_{\varepsilon}=0 \text { on } \partial F_{\varepsilon}\right\}, \quad V_{\varepsilon}^{0}=\{\varphi \in V: \operatorname{div}(\varphi)=0\},
$$

equipped with the norm $\|\nabla \varphi\|_{L^{2}\left(\Omega_{\varepsilon}\right)^{3 \times 3}}$ and the $L_{0}^{2}$ the space of functions of $L^{2}$ with null integral equipped with the norm of $L^{2}$. Let $C_{\mathrm{per}}^{\infty}(Y)$ be the space of infinitely differentiable functions in $\mathbb{R}^{3}$ that are $Y$-periodic. By $L_{\text {per }}^{2}(Y)$ (resp. $\left.H_{\text {per }}^{1}(Y)\right)$ we denote its completion in the norm $L^{2}(Y)$ (resp. $\left.H^{1}(Y)\right)$. We denote by $L_{0, \text { per }}^{2}(Y)$ the space of functions in $L_{\mathrm{per}}^{2}(Y)$ with null integral. We also define the spaces $V_{Y}$ and $V_{Y}^{0}$ are given by

$$
V_{Y}=\left\{\varphi \in H_{\mathrm{per}}^{1}\left(Y^{*}\right)^{3}: \varphi \cdot n=0 \text { on } \partial F\right\}, \quad V_{Y}^{0}=\left\{\varphi \in V_{Y}: \operatorname{div}_{y}(\varphi)=0 \text { in } Y^{*}\right\} .
$$

\section{Main results}

In this section we give the main results of this paper. First, the existence and uniqueness of solution of problem (2.15)-(2.23) is established in Theorem 3.2 and then, the homogenized model is given in Theorem 3.3. The proof of the corresponding results are given in the next section.

In order to prove, for each value of $\varepsilon$, the existence and uniqueness of solution of problem (2.15)-(2.23), instead of working directly with the classical variational formulation, we will work with an equivalent variational formulation. 
Proposition 3.1. Sufficiently regular solutions of (2.15)-(2.23) satisfy the following weak formulation:

Find $\left(u_{\varepsilon}, w_{\varepsilon}, p_{\varepsilon}\right) \in V_{\varepsilon}^{0} \times V_{\varepsilon} \times L_{0}^{2}\left(\Omega_{\varepsilon}\right)$ such that

$$
\begin{gathered}
\int_{\Omega_{\varepsilon}} \operatorname{rot}\left(u_{\varepsilon}\right) \cdot \operatorname{rot}(\varphi) d x-\int_{\Omega_{\varepsilon}} p_{\varepsilon} \operatorname{div}(\varphi) d x-2 N^{2} \int_{\Omega_{\varepsilon}} \operatorname{rot}(\varphi) \cdot w_{\varepsilon} d x \\
+2\left(\frac{1}{\alpha}-N^{2}\right) \int_{\partial F_{\varepsilon}}\left(w_{\varepsilon} \times n_{\varepsilon}\right) \cdot \varphi d \sigma=\varepsilon^{-1} \int_{\Omega_{\varepsilon}} f \cdot \varphi d x, \quad \forall \varphi \in V_{\varepsilon}, \\
\varepsilon^{2} R_{c} \int_{\Omega_{\varepsilon}} \operatorname{rot}\left(w_{\varepsilon}\right) \cdot \operatorname{rot}(\psi) d x+\varepsilon^{2} R_{c} \int_{\Omega_{\varepsilon}} \operatorname{div}\left(w_{\varepsilon}\right) \cdot \operatorname{div}(\psi) d x+4 N^{2} \int_{\Omega_{\varepsilon}} w_{\varepsilon} \cdot \psi d x \\
-2 N^{2} \int_{\Omega_{\varepsilon}} \operatorname{rot}(\psi) \cdot u_{\varepsilon} d x+2 N^{2}(\beta-1) \int_{\partial F_{\varepsilon}}\left(u_{\varepsilon} \times n_{\varepsilon}\right) \cdot \psi d \sigma=\int_{\Omega_{\varepsilon}} g \cdot \psi d x, \quad \forall \psi \in V_{\varepsilon} .
\end{gathered}
$$

We give the result of the existence and uniqueness of solution of problem (3.24)-(3.25).

Theorem 3.2. Let $\gamma=\frac{1}{\alpha}-N^{2}-N^{2} \beta$. Assume that the asymptotic regimes (2.14) and (2.18) hold. Then, for any $\alpha$ and $\beta$ satisfying

$$
\gamma^{2}<\frac{R_{c}\left(1-N^{2}\right)}{K^{2}}
$$

there exists a unique solution $\left(u_{\varepsilon}, w_{\varepsilon}, p_{\varepsilon}\right) \in V_{\varepsilon}^{0} \times V_{\varepsilon} \times L_{0}^{2}\left(\Omega_{\varepsilon}\right)$ of problem (3.24)-(3.25), with $K=C_{p t} C_{g}$ where $C_{p t}$ and $C_{g}$ are the trace and the Gaffney constants given, respectively, in Corollary 4.3 and Lemma 4.4, both placed in the next section.

We describe the asymptotic behavior of the sequences $u_{\varepsilon}, w_{\varepsilon}$ and $p_{\varepsilon}$ when $\varepsilon$ tends to zero. To do this, we take into account that the sequence of solutions $\left(u_{\varepsilon}, w_{\varepsilon}, p_{\varepsilon}\right) \in V_{\varepsilon}^{0} \times V_{\varepsilon} \times L_{0}^{2}\left(\Omega_{\varepsilon}\right)$ is not defined in a fixed domain independent of $\varepsilon$ and $a_{\varepsilon}$ but rather in a varying set $\Omega_{\varepsilon}$. Thus, in order to pass to the limit when $\varepsilon$ tends to zero, convergences in fixed Sovolev spaces (defined in $\Omega$ ) are used which require first that $\left(u_{\varepsilon}, w_{\varepsilon}, p_{\varepsilon}\right)$ be extended to the whole domain $\Omega$. Then, we define an extension $\left(U_{\varepsilon}, W_{\varepsilon}, P_{\varepsilon}\right)$ of $\left(u_{\varepsilon}, w_{\varepsilon}, p_{\varepsilon}\right)$ on $\Omega$ which coincides with $\left(u_{\varepsilon}, w_{\varepsilon}, p_{\varepsilon}\right)$ on $\Omega_{\varepsilon}$.

Theorem 3.3. Assume that the asymptotic regimes (2.14) and (2.18) and condition (3.26) hold. Then, the whole sequences of extensions $\left(\varepsilon^{-1} U_{\varepsilon}, W_{\varepsilon}\right)$ and $\varepsilon P_{\varepsilon}$ of the solution of problem (2.15)-(2.23) converge weakly to $(u, w)$ in $L^{2}(\Omega)^{3} \times L^{2}(\Omega)^{3}$ and strongly to $p$ in $L^{2}(\Omega)$ respectively. Moreover, it holds

$$
u(x)=K^{(1)}(f(x)-\nabla p(x))+K^{(2)} g(x), \quad w(x)=L^{(1)}(f(x)-\nabla p(x))+L^{(2)} g(x) \quad \text { in } \Omega,
$$

and moreover, $p \in H^{1}(\Omega) \cap L_{0}^{2}(\Omega)$ is the unique solution of the Darcy equation

$$
\left\{\begin{aligned}
\operatorname{div}\left(K^{(1)}(f(x)-\nabla p(x))+K^{(2)} g(x)\right)=0 & \text { in } \Omega, \\
\left(K^{(1)}(f(x)-\nabla p(x))+K^{(2)} g(x)\right) \cdot n=0 & \text { on } \partial \Omega .
\end{aligned}\right.
$$

The matrix coefficients $K^{(k)}, L^{(k)} \in \mathbb{R}^{3 \times 3}, k=1,2$, where $K^{(1)}$ is positive definite, are given by

$$
K_{i j}^{(k)}=\int_{Y^{*}} u_{j}^{i, k}(y) d y, \quad L_{i j}^{(k)}=\int_{Y^{*}} w_{j}^{i, k}(y) d y, \quad i, j=1,2,3,
$$


with $\left(u^{i, k}, w^{i, k}, \pi^{i, k}\right) \in V_{Y}^{0} \times V_{Y} \times L_{0, \mathrm{per}}^{2}\left(Y^{*}\right), k=1,2, i=1,2,3$, the unique solution of local micropolar problem

$$
\left\{\begin{aligned}
-\Delta_{y} u^{i, k}+\nabla_{y} \pi^{i, k}-2 N^{2} \operatorname{rot}_{y}\left(w^{i, k}\right)=e_{i} \delta_{1 k} & \text { in } Y^{*}, \\
-R_{c} \Delta_{y} w^{i, k}+4 N^{2} w^{i, k}-2 N^{2} \operatorname{rot}_{y}\left(u^{i, k}\right)=e_{i} \delta_{2 k} & \text { in } Y^{*}, \\
\operatorname{div}_{y}\left(u^{i, k}\right)=0 & \text { in } Y^{*}, \\
w^{i, k} \times n=\frac{\alpha}{2} \operatorname{rot}\left(u^{i, k}\right) \times n & \text { on } \partial F, \\
\operatorname{rot}\left(w^{i, k}\right) \times n=\frac{2 N^{2}}{R_{c}} \beta\left(u^{i, k} \times n\right) & \text { on } \partial F, \\
u^{i, k} \cdot n=0 & \text { on } \partial F, \\
w^{i, k} \cdot n=0 & \text { on } \partial F .
\end{aligned}\right.
$$

\section{Proof of the results of Section 3}

Proof of Proposition 3.1. First, from (2.16) and the boundary conditions (2.21) and (2.22), solutions of (2.15)-(2.23) are in $V_{\varepsilon}^{0} \times V_{\varepsilon}$.

Next, to obtain (3.24), we take $\varphi \in V_{\varepsilon}$ as test function in (2.15) and using (2.11) and (2.13) we get

$$
\begin{aligned}
& \int_{\Omega_{\varepsilon}} \operatorname{rot}\left(u_{\varepsilon}\right) \cdot \operatorname{rot}(\varphi) d x-\int_{\Omega_{\varepsilon}} p_{\varepsilon} \operatorname{div}(\varphi) d x+\int_{\partial F_{\varepsilon}}\left(\operatorname{rot}\left(u_{\varepsilon}\right) \times n_{\varepsilon}\right) \cdot \varphi d \sigma \\
& -2 N^{2} \int_{\Omega_{\varepsilon}} \operatorname{rot}(\varphi) \cdot w_{\varepsilon} d x-2 N^{2} \int_{\partial F_{\varepsilon}}\left(w_{\varepsilon} \times n_{\varepsilon}\right) \cdot \varphi d \sigma=\varepsilon^{-1} \int_{\Omega_{\varepsilon}} f \cdot \varphi d x .
\end{aligned}
$$

Thus, taking into account the boundary condition (2.19), we derive equation (3.24).

Finally, to obtain (3.25), we take $\psi \in V_{\varepsilon}$ as test function in (2.17) and proceeding as above we get

$$
\begin{aligned}
& \varepsilon^{2} R_{c} \int_{\Omega_{\varepsilon}} \operatorname{rot}\left(w_{\varepsilon}\right) \cdot \operatorname{rot}(\psi) d x+\varepsilon^{2} R_{c} \int_{\Omega_{\varepsilon}} \operatorname{div}\left(w_{\varepsilon}\right) \operatorname{div}(\psi) d x+\varepsilon^{2} R_{c} \int_{\partial F_{\varepsilon}}\left(\operatorname{rot}\left(w_{\varepsilon}\right) \times n_{\varepsilon}\right) \cdot \psi d \sigma \\
& +4 N^{2} \int_{\Omega_{\varepsilon}} w_{\varepsilon} \cdot \psi d x-2 N^{2} \int_{\Omega_{\varepsilon}} \operatorname{rot}(\psi) \cdot u_{\varepsilon} d x-2 N^{2} \int_{\partial F_{\varepsilon}}\left(u_{\varepsilon} \times n_{\varepsilon}\right) \cdot \psi d \sigma=\int_{\Omega_{\varepsilon}} g \cdot \psi d x .
\end{aligned}
$$

Taking into account the boundary condition (2.20), we derive equation (3.25).

Before proving the result concerning existence and uniqueness of solution, we give several technical lemmas which will also be used to obtain a priori estimates of the solution. First, we recall a result about a trace result on the boundary of the obstacles $\partial F_{\varepsilon}$ whose proof can be found in $[2,5,19]$.

Lemma 4.1. There exists a positive constant $C_{t}$ independent of $\varepsilon$, such that for every $v \in H^{1}\left(\Omega_{\varepsilon}\right)^{3}$,

$$
\|v\|_{L^{2}\left(\partial F_{\varepsilon}\right)^{3}}^{2} \leq \varepsilon^{-1} C_{t}\left(\|v\|_{L^{2}\left(\Omega_{\varepsilon}\right)^{3}}^{2}+\varepsilon^{2}\|D v\|_{L^{2}\left(\Omega_{\varepsilon}\right)^{3 \times 3}}^{2}\right) .
$$

We note that the fact that the normal component of the function is equal zero on $\partial F_{\varepsilon}$ is not used in the previous estimate, so it holds true in a more general context. However, the next inequalities in $\Omega_{\varepsilon}$ makes use of this condition and will be also used for obtaining a priori estimates for the velocity and microrotation. Thus, we first recall the version of Poincaré's inequality given in $[2,14]$.

Lemma 4.2. There exists a positive constant $C_{p}$, independent of $\varepsilon$, such that for every $v \in V_{\varepsilon}$,

$$
\|v\|_{L^{2}\left(\Omega_{\varepsilon}\right)^{3}} \leq \varepsilon C_{p}\|D v\|_{L^{2}\left(\Omega_{\varepsilon}\right)^{3 \times 3}} .
$$

Moreover, there exists a positive constant $C$, independent of $\varepsilon$, such that for every $v \in H^{1}(\Omega)^{3}$ with $v \cdot n_{\varepsilon}=0$ on $\partial F_{\varepsilon}$,

$$
\|v\|_{L^{2}(\Omega)^{3}} \leq \varepsilon C\|D v\|_{L^{2}(\Omega)^{3 \times 3}} .
$$


As a consequence of previous results, we deduce the following result.

Corollary 4.3. For every $v \in V_{\varepsilon}$, the following estimate holds

$$
\|v\|_{L^{2}\left(\partial F_{\varepsilon}\right)^{3}} \leq\left(\varepsilon C_{p t}\right)^{\frac{1}{2}}\|D v\|_{L^{2}\left(\Omega_{\varepsilon}\right)^{3 \times 3}},
$$

where the positive constant $C_{p t}=C_{t}^{2}\left(C_{p}^{2}+1\right)^{2}$ with $C_{t}$ given in (4.30) and $C_{p}$ given in (4.31).

We also give an estimate of the derivative in terms of the divergence and the rotational, necessary to prove the coercivity of the variational formulation. It has different names in the literature, e.g. Gaffney's, Maxwell's or Friedrichs' inequality, see [4, 13, 24, 28].

Lemma 4.4. There exists a positive constant $C_{g}$, independent of $\varepsilon$, such that for every $v \in V_{\varepsilon}$,

$$
\|D v\|_{L^{2}\left(\Omega_{\varepsilon}\right)^{3 \times 3}}^{2} \leq C_{g}\left(\|\operatorname{div}(v)\|_{L^{2}\left(\Omega_{\varepsilon}\right)}^{2}+\|\operatorname{rot}(v)\|_{L^{2}\left(\Omega_{\varepsilon}\right)^{3}}^{2}\right) .
$$

Moreover, for every $v \in V_{\varepsilon}^{0}$ it holds

$$
\|D v\|_{L^{2}\left(\Omega_{\varepsilon}\right)^{3 \times 3}} \leq C_{g}\|\operatorname{rot}(v)\|_{L^{2}\left(\Omega_{\varepsilon}\right)^{3}} .
$$

Proof. For every function $v \in H^{1}\left(Y_{k, 1}^{*}\right)^{3}$ such that $v \cdot n=0$ on $\partial F_{k, 1}$, using Theorem IV.4.8. in [13] (see also Chapter 7, Lema 6.1 in [24]), the fact that $Y_{k, 1}^{*}$ is simply connected and that the boundary $\partial F_{k, 1}$ is $C^{1,1}$, we have, for every $k \in \mathbb{Z}^{3}$, that

$$
\|D v\|_{L^{2}\left(Y_{k, 1}^{*}\right)^{3 \times 3}}^{2} \leq C_{g}\left(\|\operatorname{div}(v)\|_{L^{2}\left(Y_{k, 1}^{*}\right)}^{2}+\|\operatorname{rot}(v)\|_{L^{2}\left(Y_{k, 1}^{*}\right)^{3}}^{2}\right),
$$

where the positive constant $C_{g}$ only depends on $Y_{k, 1}^{*}$.

By the change of variable

$$
y=\frac{x}{\varepsilon}, \quad d y=\varepsilon^{-3} d x, \quad \partial_{y}=\varepsilon \partial_{x},
$$

we rescale (4.36) from $Y_{k, 1}^{*}$ to $Y_{k, \varepsilon}^{*}$ and from $F_{k, 1}$ to $F_{k, \varepsilon}$. This yields

$$
\|D v\|_{L^{2}\left(Y_{k, \varepsilon}^{*}\right)^{3 \times 3}}^{2} \leq C_{g}\left(\|\operatorname{div}(v)\|_{L^{2}\left(Y_{k, \varepsilon}^{*}\right)}^{2}+\|\operatorname{rot}(v)\|_{L^{2}\left(Y_{k, \varepsilon}^{*}\right)^{3}}^{2}\right) .
$$

Summing the inequalities, for every $k \in \mathcal{K}_{\varepsilon}$, gives (4.34) (estimate (4.35) is straightforward).

In fact, we must consider separately the periods containing a portion of $\partial \Omega$, but they yield at a distance $O(\varepsilon)$ of $\partial \Omega$, where $v$ is zero, and then the corresponding inequality is immediately obtained.

Proof of Theorem 3.2. To prove, for each value of $\varepsilon>0$, the existence and uniqueness of a weak solution $\left(u_{\varepsilon}, w_{\varepsilon}, p_{\varepsilon}\right)$ of problem (3.24)-(3.25), we shall apply classical results given in [27]. To this purpose, we introduce the following equivalent mixed variational form:

Find $\left(u_{\varepsilon}, w_{\varepsilon}, p_{\varepsilon}\right) \in V_{\varepsilon} \times V_{\varepsilon} \in L_{0}^{2}\left(\Omega_{\varepsilon}\right)$ such that

$$
\begin{array}{rlrlrl}
\mathcal{A}_{\varepsilon}\left(u_{\varepsilon}, w_{\varepsilon} ; \varphi, \psi\right)+\mathcal{B}_{\varepsilon}\left((\varphi, \psi), p_{\varepsilon}\right) & = & \mathcal{L}_{\varepsilon}(\varphi, \psi) & & \forall(\varphi, \psi) \in V_{\varepsilon} \times V_{\varepsilon}, \\
\mathcal{B}_{\varepsilon}\left(\left(u_{\varepsilon}, w_{\varepsilon}\right), q_{\varepsilon}\right) & =0 & & \forall q_{\varepsilon} \in L_{0}^{2}\left(\Omega_{\varepsilon}\right),
\end{array}
$$

where

$$
\begin{aligned}
\mathcal{A}_{\varepsilon}\left(u_{\varepsilon}, w_{\varepsilon} ; \varphi, \psi\right)= & \int_{\Omega_{\varepsilon}} \operatorname{rot}\left(u_{\varepsilon}\right) \cdot \operatorname{rot}(\varphi) d x-2 N^{2} \int_{\Omega_{\varepsilon}} \operatorname{rot}(\varphi) \cdot w_{\varepsilon} d x \\
& +\varepsilon^{2} R_{c} \int_{\Omega_{\varepsilon}} \operatorname{rot}\left(w_{\varepsilon}\right) \cdot \operatorname{rot}(\psi) d x+\varepsilon^{2} R_{c} \int_{\Omega_{\varepsilon}} \operatorname{div}\left(w_{\varepsilon}\right) \cdot \operatorname{div}(\psi) d x \\
& -2 N^{2} \int_{\Omega_{\varepsilon}} \operatorname{rot}(\psi) \cdot u_{\varepsilon} d x+4 N^{2} \int_{\Omega_{\varepsilon}} w_{\varepsilon} \cdot \psi d x \\
& +2\left(\frac{1}{\alpha}-N^{2}\right) \int_{\partial F_{\varepsilon}}\left(w_{\varepsilon} \times n_{\varepsilon}\right) \cdot \varphi d \sigma+2 N^{2}(\beta-1) \int_{\partial F_{\varepsilon}}\left(u_{\varepsilon} \times n_{\varepsilon}\right) \cdot \psi d \sigma,
\end{aligned}
$$


and

$$
\mathcal{B}_{\varepsilon}\left((\varphi, \psi), p_{\varepsilon}\right)=-\int_{\Omega_{\varepsilon}} p_{\varepsilon} \operatorname{div}(\varphi) d x, \quad \mathcal{L}_{\varepsilon}(\varphi, \psi)=\varepsilon^{-1} \int_{\Omega_{\varepsilon}} f \cdot \varphi d x+\int_{\Omega_{\varepsilon}} g \cdot \psi d x .
$$

It is easy to prove that the bilinear form $\mathcal{A}_{\varepsilon}, \mathcal{B}_{\varepsilon}$ and $\mathcal{L}_{\varepsilon}$ are continuous bilinear forms on $\left(V_{\varepsilon} \times V_{\varepsilon}\right)^{2}, V_{\varepsilon} \times V_{\varepsilon} \times L_{0}^{2}\left(\Omega_{\varepsilon}\right)$ and $V_{\varepsilon} \times V_{\varepsilon}$ respectively. Denoting

$$
\|(\varphi, \psi)\|_{V_{\varepsilon}^{0} \times V_{\varepsilon}}=\left(\|D \varphi\|_{L^{2}\left(\Omega_{\varepsilon}\right)^{3 \times 3}}^{2}+\|D \psi\|_{L^{2}\left(\Omega_{\varepsilon}\right)^{3 \times 3}}^{2}\right)^{\frac{1}{2}} .
$$

classical existence conditions for such a problem [27, Theorem 4.1] are the coerciveness of the form $\mathcal{A}_{\varepsilon}$ on the subspace $\left(V_{\varepsilon}^{0} \times V_{\varepsilon}\right)^{2}$ and the inf-sup condition.

First, we prove that $\mathcal{A}_{\varepsilon}$ is coercive on $\left(V_{\varepsilon}^{0} \times V_{\varepsilon}\right)^{2}$, i.e. that there exists $\eta>0$ such that

$$
\mathcal{A}_{\varepsilon}(\varphi, \psi ; \varphi, \psi) \geq \eta\|(\varphi, \psi)\|_{V_{\varepsilon}^{0} \times V_{\varepsilon}}^{2} .
$$

To do this, let us derive another equivalent expression for $\mathcal{A}_{\varepsilon}$. By using $(2.13)$ applied to $\int_{\Omega_{\varepsilon}} \operatorname{rot}(\psi) \cdot u_{\varepsilon} d x$ and using that $\int_{\partial F_{\varepsilon}}\left(\psi \times n_{\varepsilon}\right) \cdot u_{\varepsilon} d \sigma=-\int_{\partial F_{\varepsilon}}\left(u_{\varepsilon} \times n_{\varepsilon}\right) \cdot \psi d \sigma$, we have that $\mathcal{A}_{\varepsilon}$ defined in (4.38) has the following expression

$$
\begin{aligned}
\mathcal{A}_{\varepsilon}\left(u_{\varepsilon}, w_{\varepsilon} ; \varphi, \psi\right)= & \int_{\Omega_{\varepsilon}} \operatorname{rot}\left(u_{\varepsilon}\right) \cdot \operatorname{rot}(\varphi) d x-2 N^{2} \int_{\Omega_{\varepsilon}} \operatorname{rot}(\varphi) \cdot w_{\varepsilon} d x \\
& +\varepsilon^{2} R_{c} \int_{\Omega_{\varepsilon}} \operatorname{rot}\left(w_{\varepsilon}\right) \cdot \operatorname{rot}(\psi) d x+\varepsilon^{2} R_{c} \int_{\Omega_{\varepsilon}} \operatorname{div}\left(w_{\varepsilon}\right) \cdot \operatorname{div}(\psi) d x \\
& -2 N^{2} \int_{\Omega_{\varepsilon}} \operatorname{rot}\left(u_{\varepsilon}\right) \cdot \psi d x+4 N^{2} \int_{\Omega_{\varepsilon}} w_{\varepsilon} \cdot \psi d x+2 \gamma \int_{\partial F_{\varepsilon}}\left(w_{\varepsilon} \times n_{\varepsilon}\right) \cdot \varphi d \sigma .
\end{aligned}
$$

Then, we have

$$
\begin{aligned}
\mathcal{A}_{\varepsilon}(\varphi, \psi ; \varphi, \psi)= & \int_{\Omega_{\varepsilon}}|\operatorname{rot}(\varphi)|^{2} d x-4 N^{2} \int_{\Omega_{\varepsilon}} \operatorname{rot}(\varphi) \cdot \psi d x+\varepsilon^{2} R_{c} \int_{\Omega_{\varepsilon}}|\operatorname{rot}(\psi)|^{2} d x \\
& +\varepsilon^{2} R_{c} \int_{\Omega_{\varepsilon}}|\operatorname{div}(\psi)|^{2} d x+4 N^{2} \int_{\Omega_{\varepsilon}}|\psi|^{2} d x+2 \gamma \int_{\partial F_{\varepsilon}}\left(\psi \times n_{\varepsilon}\right) \cdot \varphi d \sigma .
\end{aligned}
$$

From the Cauchy-Schwartz inequality and (4.33), we get

$$
\int_{\partial F_{\varepsilon}}\left(\psi \times n_{\varepsilon}\right) \cdot \varphi d \sigma \leq \varepsilon C_{p t}\|D \psi\|_{L^{2}\left(\Omega_{\varepsilon}\right)^{3 \times 3}}\|D \varphi\|_{L^{2}\left(\Omega_{\varepsilon}\right)^{3 \times 3}},
$$

and by using (4.34) and (4.35), we deduce

$$
\begin{aligned}
\mathcal{A}_{\varepsilon}(\varphi, \psi ; \varphi, \psi) \geq & \frac{1}{C_{g}}\|D \varphi\|_{L^{2}\left(\Omega_{\varepsilon}\right)^{3 \times 3}}^{2}-4 \sqrt{3} N^{2}\|D \varphi\|_{L^{2}\left(\Omega_{\varepsilon}\right)^{3 \times 3}}\|\psi\|_{L^{2}\left(\Omega_{\varepsilon}\right)^{3}} \\
& +\varepsilon^{2} \frac{R_{c}}{C_{g}}\|D \psi\|_{L^{2}\left(\Omega_{\varepsilon}\right)^{3 \times 3}}^{2}+4 N^{2}\|\psi\|_{L^{2}\left(\Omega_{\varepsilon}\right)^{3}}^{2} \\
& -2|\gamma| \varepsilon C_{p t}\|D \varphi\|_{L^{2}\left(\Omega_{\varepsilon}\right)^{3 \times 3}}\|D \psi\|_{L^{2}\left(\Omega_{\varepsilon}\right)^{3 \times 3}} .
\end{aligned}
$$

Now, by condition (3.26), there exists $c_{1}>0$ satisfying

$$
\frac{|\gamma| C_{p t} C_{g}^{2}}{R_{c}}<c_{1}<\frac{1-N^{2}}{|\gamma| C_{p t}}
$$

and by Young's inequality,

$$
\|D \varphi\|_{L^{2}\left(\Omega_{\varepsilon}\right)^{3 \times 3}}\|D \psi\|_{L^{2}\left(\Omega_{\varepsilon}\right)^{3 \times 3}} \leq \frac{c_{1}}{2 \varepsilon C_{g}}\|D \varphi\|_{L^{2}\left(\Omega_{\varepsilon}\right)^{3 \times 3}}^{2}+\frac{\varepsilon C_{g}}{2 c_{1}}\|D \psi\|_{L^{2}\left(\Omega_{\varepsilon}\right)^{3 \times 3}}^{2} .
$$


Introducing a real number $c_{2}$ satisfying $0<c_{2}<\min \left\{1,1 /\left(3 C_{g}\right)\right\}$, and such that

$$
c_{1}<\frac{1-\frac{N^{2}}{c_{2}}}{|\gamma| C_{p t}} \quad\left(\text { and so } \frac{|\gamma| C_{p t} C_{g}^{2}}{R_{c}}<c_{1}<\frac{1-\frac{N^{2}}{c_{2}}}{|\gamma| C_{p t}}<\frac{1-N^{2}}{|\gamma| C_{p t}}\right),
$$

we also have by Young's inequality

$$
\|D \varphi\|_{L^{2}\left(\Omega_{\varepsilon}\right)^{3 \times 3}}\|\psi\|_{L^{2}\left(\Omega_{\varepsilon}\right)^{3}} \leq \frac{1}{4 \sqrt{3} c_{2} C_{g}}\|D \varphi\|_{L^{2}\left(\Omega_{\varepsilon}\right)^{3 \times 3}}^{2}+\sqrt{3} c_{2} C_{g}\|\psi\|_{L^{2}\left(\Omega_{\varepsilon}\right)^{3}}^{2} .
$$

Going back to estimate (4.41), we obtain

$$
\mathcal{A}_{\varepsilon}(\varphi, \psi ; \varphi, \psi) \geq A\|D \varphi\|_{L^{2}\left(\Omega_{\varepsilon}\right)^{3 \times 3}}^{2}+\varepsilon^{2} B\|D \psi\|_{L^{2}\left(\Omega_{\varepsilon}\right)^{3 \times 3}}^{2}+4 N^{2}\left(1-3 c_{2} C_{g}\right)\|\psi\|_{L^{2}\left(\Omega_{\varepsilon}\right)^{3}}^{2},
$$

where $A, B$ are defined as follows

$$
A=\frac{1}{C_{g}}\left(1-\frac{N^{2}}{c_{2}}-|\gamma| C_{p t} c_{1}\right), \quad B=\frac{1}{C_{g}}\left(R_{c}-\frac{|\gamma| C_{p t}}{c_{1}} C_{g}^{2}\right) .
$$

Thus, we have

$$
\mathcal{A}_{\varepsilon}(\varphi, \psi ; \varphi, \psi) \geq A\|D \varphi\|_{L^{2}\left(\Omega_{\varepsilon}\right)^{3 \times 3}}^{2}+\varepsilon^{2} B\|D \psi\|_{L^{2}\left(\Omega_{\varepsilon}\right)^{3 \times 3}}^{2},
$$

which proves that (4.39) holds.

Next, we prove the inf-sup condition, i.e. that there exists $\delta>0$ such that

$$
\inf _{q_{\varepsilon} \in L_{0}^{2}\left(\Omega_{\varepsilon}\right)} \sup _{\left(u_{\varepsilon}, \psi\right) \in V_{\varepsilon}^{2}} \frac{\mathcal{B}_{\varepsilon}\left(\left(u_{\varepsilon}, \psi\right), q_{\varepsilon}\right)}{\left\|\left(u_{\varepsilon}, \psi\right)\right\|_{V_{\varepsilon}^{2}}\left\|q_{\varepsilon}\right\|_{L_{0}^{2}\left(\Omega_{\varepsilon}\right)}} \geq \delta .
$$

Let $(\varphi, \psi)$ belong to $V_{\varepsilon} \times V_{\varepsilon}$, and $q_{\varepsilon}$ to $L_{0}^{2}\left(\Omega_{\varepsilon}\right)$, we obtain

$$
H_{0}^{1}\left(\Omega_{\varepsilon}\right)^{3} \times\{0\} \subseteq V_{\varepsilon} \times V_{\varepsilon},
$$

so that

$$
\sup _{(\varphi, \psi) \in V_{\varepsilon} \times V_{\varepsilon}} \frac{\int_{\Omega_{\varepsilon}} \operatorname{div} \varphi q_{\varepsilon} d x}{\left(\|\varphi\|_{V_{\varepsilon}}^{2}+\|\varphi\|_{V_{\varepsilon}}^{2}\right)^{\frac{1}{2}}} \geq \sup _{(\varphi, 0) \in H_{0}^{1}\left(\Omega_{\varepsilon}\right)^{3} \times\{0\}} \frac{\int_{\Omega_{\varepsilon}} \operatorname{div} \varphi q_{\varepsilon} d x}{\|D \varphi\|_{L^{2}\left(\Omega_{\varepsilon}\right)^{3 \times 3}}}=\sup _{\varphi \in H_{0}^{1}\left(\Omega_{\varepsilon}\right)^{3}} \frac{\int_{\Omega_{\varepsilon}} \operatorname{div} \varphi q_{\varepsilon} d x}{\|D \varphi\|_{L^{2}\left(\Omega_{\varepsilon}\right)^{3 \times 3}}} .
$$

According to the inverse of the divergence operator in perforated domains, see for example [26], for a given $q_{\varepsilon} \in$ $L_{0}^{2}\left(\Omega_{\varepsilon}\right)$, there exists $v_{\varepsilon}\left[q_{\varepsilon}\right] \in H_{0}^{1}\left(\Omega_{\varepsilon}\right)^{3}$ such that $\operatorname{div} v_{\varepsilon}\left[q_{\varepsilon}\right]=q_{\varepsilon}$ in $\Omega_{\varepsilon}$ and $\left\|D v_{\varepsilon}\left[q_{\varepsilon}\right]\right\|_{L^{2}\left(\Omega_{\varepsilon}\right)^{3 \times 3}} \leq C\left\|q_{\varepsilon}\right\|_{L^{2}\left(\Omega_{\varepsilon}\right)}$ for some constant $C>0$ independent of $\varepsilon$. Choosing $\varphi=v_{\varepsilon}\left[q_{\varepsilon}\right]$, we get

$$
\sup _{(\varphi, \psi) \in V_{\varepsilon} \times V_{\varepsilon}} \frac{\int_{\Omega_{\varepsilon}} \operatorname{div} \varphi q_{\varepsilon} d x}{\left(\|\varphi\|_{V_{\varepsilon}}^{2}+\|\varphi\|_{V_{\varepsilon}}^{2}\right)^{\frac{1}{2}}} \geq \frac{\int_{\Omega_{\varepsilon}} \operatorname{div} v_{\varepsilon}\left[q_{\varepsilon}\right] q_{\varepsilon} d x}{\left\|D v_{\varepsilon}\left[q_{\varepsilon}\right]\right\|_{L^{2}\left(\Omega_{\varepsilon}\right)^{3 \times 3}}}=\frac{\left\|q_{\varepsilon}\right\|_{L^{2}\left(\Omega_{\varepsilon}\right)}^{2}}{\left\|D v_{\varepsilon}\left[q_{\varepsilon}\right]\right\|_{L^{2}\left(\Omega_{\varepsilon}\right)^{3 \times 3}}} \geq \frac{1}{C}\left\|q_{\varepsilon}\right\|_{L^{2}\left(\Omega_{\varepsilon}\right)} .
$$

This ends the proof.

A priori estimates. We establish sharp a priori estimates of the solution in $\Omega_{\varepsilon}$ and also for extended solution to $\Omega$, which is independent of $\varepsilon$, introducing suitable extension operators.

We give the a priori estimates for velocity and microrotation in $\Omega_{\varepsilon}$.

Lemma 4.5. Assume that the asymptotic regimes (2.14) and (2.18) and condition (3.26) hold. Then there exists a positive constant $C$, independent of $\varepsilon$, such that the following estimates for the velocity and microrotation hold

$$
\begin{gathered}
\varepsilon^{-1}\left\|u_{\varepsilon}\right\|_{L^{2}\left(\Omega_{\varepsilon}\right)^{3}}+\left\|D u_{\varepsilon}\right\|_{L^{2}\left(\Omega_{\varepsilon}\right)^{3 \times 3}} \leq C, \\
\left\|w_{\varepsilon}\right\|_{L^{2}\left(\Omega_{\varepsilon}\right)^{3}}+\varepsilon\left\|D w_{\varepsilon}\right\|_{L^{2}\left(\Omega_{\varepsilon}\right)^{3 \times 3}} \leq C .
\end{gathered}
$$


Proof. To obtain estimates of velocity and microrotation we consider $(\varphi, \psi)=\left(u_{\varepsilon}, w_{\varepsilon}\right)$ as test functions in the weak formulation (3.24)-(3.25) and use (4.43) and Young's inequality to obtain

$$
\begin{aligned}
A \| D & u_{\varepsilon}\left\|_{L^{2}\left(\Omega_{\varepsilon}\right)^{3 \times 3}}^{2}+\varepsilon^{2} B\right\| D w_{\varepsilon} \|_{L^{2}\left(\Omega_{\varepsilon}\right)^{3 \times 3}}^{2} \\
& \leq C_{p}\|f\|_{L^{2}(\Omega)^{3}}\left\|D u_{\varepsilon}\right\|_{L^{2}\left(\Omega_{\varepsilon}\right)^{3 \times 3}}+\varepsilon C_{p}\|g\|_{L^{2}(\Omega)^{3}}\left\|D w_{\varepsilon}\right\|_{L^{2}\left(\Omega_{\varepsilon}\right)^{3 \times 3}} \\
& \leq \frac{C_{p}^{2}}{2 A}\|f\|_{L^{2}(\Omega)^{3}}^{2}+\frac{A}{2}\left\|D u_{\varepsilon}\right\|_{L^{2}\left(\Omega_{\varepsilon}\right)^{3 \times 3}}^{2}+\frac{C_{p}^{2}}{2 B}\|g\|_{L^{2}(\Omega)^{3}}^{2}+\frac{\varepsilon^{2} B}{2}\left\|D w_{\varepsilon}\right\|_{L^{2}\left(\Omega_{\varepsilon}\right)^{3 \times 3}}^{2} .
\end{aligned}
$$

Then we have,

$$
\frac{A}{2}\left\|D u_{\varepsilon}\right\|_{L^{2}\left(\Omega_{\varepsilon}\right)^{3 \times 3}}^{2}+\varepsilon^{2} \frac{B}{2}\left\|D w_{\varepsilon}\right\|_{L^{2}\left(\Omega_{\varepsilon}\right)^{3 \times 3}}^{2} \leq \frac{C_{p}^{2}}{2 A}\|f\|_{L^{2}(\Omega)^{3}}^{2}+\frac{C_{p}^{2}}{2 B}\|g\|_{L^{2}(\Omega)^{3}}^{2} .
$$

Since $A$ and $B$ are bounded, we deduce than the right hand side of the previous inequality is bounded by a certain positive constant $C$ independent of $\varepsilon$, which implies the following estimates in $\Omega_{\varepsilon}$

$$
\left\|D u_{\varepsilon}\right\|_{L^{2}\left(\Omega_{\varepsilon}\right)^{3 \times 3}} \leq C, \quad\left\|D w_{\varepsilon}\right\|_{L^{2}\left(\Omega_{\varepsilon}\right)^{3 \times 3}} \leq C \varepsilon^{-1} .
$$

This and Poincaré's inequality (4.31) give the estimate for $u_{\varepsilon}$ and $w_{\varepsilon}$ respectively.

Since the solution $\left(u_{\varepsilon}, w_{\varepsilon}\right)$ of problem (2.15)-(2.23) is defined only in $\Omega_{\varepsilon}$, we need to extend them to the whole domain $\Omega$. If we had considered the micropolar equations with Dirichlet boundary condition on the obstacles, the velocity and microrotation would be extended by zero in the obstacles. However, we need another kind of extension for the case in which the velocity and microrotation are non-zero on the obstacles. Thus, we introduce an extension operator which is classical in the homogenization literature, see [2, 20, 21, 32].

Lemma 4.6. There exists an extension operator $\Pi_{\varepsilon} \in \mathcal{L}\left(H^{1}\left(\Omega_{\varepsilon}\right)^{3} ; H_{0}^{1}(\Omega)^{3}\right)$ and a positive constant $C$, independent of $\varepsilon$, such that

$$
\begin{gathered}
\Pi_{\varepsilon} v(x)=v(x), \quad \text { if } x \in \Omega_{\varepsilon}, \\
\left\|D \Pi_{\varepsilon} v\right\|_{L^{2}(\Omega)^{3 \times 3}} \leq C\|D v\|_{L^{2}\left(\Omega_{\varepsilon}\right)^{3 \times 3}}, \quad \forall v \in H^{1}\left(\Omega_{\varepsilon}\right)^{3} .
\end{gathered}
$$

Taking into account the extension $\Pi_{\varepsilon}$, we denote by $U_{\varepsilon}$ the extension $\Pi_{\varepsilon} u_{\varepsilon}$ of the velocity $u_{\varepsilon}$, and by $W_{\varepsilon}$ the extension $\Pi_{\varepsilon} w_{\varepsilon}$ of the microrotation $w_{\varepsilon}$. Next, we get the following uniform estimates in $\Omega$ as consequence of Lemmas 4.5 and 4.6 .

Corollary 4.7. Assume that the asymptotic regimes (2.14) and (2.18) and condition (3.26) hold. Then there exists a positive constant $C$, independent of $\varepsilon$, such that the following estimates for the extensions of velocity and microrotation hold

$$
\begin{gathered}
\varepsilon^{-1}\left\|U_{\varepsilon}\right\|_{L^{2}(\Omega)^{3}}+\left\|D U_{\varepsilon}\right\|_{L^{2}(\Omega)^{3 \times 3}} \leq C, \\
\left\|W_{\varepsilon}\right\|_{L^{2}(\Omega)^{3}}+\varepsilon\left\|D W_{\varepsilon}\right\|_{L^{2}(\Omega)^{3 \times 3}} \leq C .
\end{gathered}
$$

Now, we recall two important results from [32] which are concerned with the extension of the pressure $p_{\varepsilon}$ to the whole domain $\Omega$. First, we define a restriction operator $R_{\varepsilon}$ from $H_{0}^{1}(\Omega)^{3}$ into $H_{0}^{1}\left(\Omega_{\varepsilon}\right)^{3}$ and then, we extend the gradient of the pressure by duality in $H^{-1}(\Omega)^{3}$.

Lemma 4.8. There exists a restriction operator $R_{\varepsilon}$ acting from $H_{0}^{1}(\Omega)^{3}$ into $H_{0}^{1}\left(\Omega_{\varepsilon}\right)^{3}$ such that

1. $v \in H_{0}^{1}\left(\Omega_{\varepsilon}\right)^{3} \Rightarrow R_{\varepsilon} v=v$ in $\Omega_{\varepsilon}$ (elements of $H_{0}^{1}\left(\Omega_{\varepsilon}\right)$ are continuated by 0 to $\Omega$ ).

2. $\operatorname{div}(v)=0$ on $\Omega \Rightarrow \operatorname{div}\left(R_{\varepsilon} v\right)=0$ in $\Omega_{\varepsilon}$.

3. There exists a positive constant $C$, independent of $\varepsilon$, such that

$$
\left\|R_{\varepsilon} v\right\|_{L^{2}\left(\Omega_{\varepsilon}\right)^{3}}+\varepsilon\left\|D R_{\varepsilon} v\right\|_{L^{2}\left(\Omega_{\varepsilon}\right)^{3 \times 3}} \leq C\left(\|v\|_{L^{2}(\Omega)}+\varepsilon\|D v\|_{L^{2}(\Omega)^{3 \times 3}}\right) \leq C\|v\|_{H_{0}^{1}(\Omega)^{3}} .
$$


Lemma 4.9. Let $q_{\varepsilon}$ be a function in $L_{0}^{2}\left(\Omega_{\varepsilon}\right)$. There exists a unique function $Q_{\varepsilon} \in L_{0}^{2}(\Omega)$ which satisfies the following equality

$$
\left\langle\nabla Q_{\varepsilon}, v\right\rangle_{H^{-1}, H_{0}^{1}(\Omega)}=\left\langle\nabla q_{\varepsilon}, R_{\varepsilon} v\right\rangle_{H^{-1}, H_{0}^{1}\left(\Omega_{\varepsilon}\right)}, \quad \text { for every } v \in H_{0}^{1}(\Omega)^{3} .
$$

We denote by $P_{\varepsilon}$ the extension of the pressure $p_{\varepsilon}$ obtained by applying Lemma 4.9 and give the following result.

Lemma 4.10. Assume that the asymptotic regimes (2.14) and (2.18) and condition (3.26) hold. Then there exists a positive constant $C$ independent of $\varepsilon$, such that the following estimate holds

$$
\varepsilon\left\|P_{\varepsilon}\right\|_{L^{2}(\Omega)}+\varepsilon\left\|\nabla P_{\varepsilon}\right\|_{H^{-1}(\Omega)^{3}} \leq C .
$$

Proof. From the definition (4.50) of the extension $P_{\varepsilon}$ and the variational formulation (3.24), we get

$$
\begin{aligned}
\left\langle\nabla P_{\varepsilon}, v\right\rangle_{H^{-1}, H_{0}^{1}(\Omega)}= & -\int_{\Omega_{\varepsilon}} \operatorname{rot}\left(u_{\varepsilon}\right) \cdot \operatorname{rot}\left(R_{\varepsilon} v\right) d x+2 N^{2} \int_{\Omega_{\varepsilon}} \operatorname{rot}\left(R_{\varepsilon} v\right) \cdot w_{\varepsilon} d x \\
& -2\left(\frac{1}{\alpha}-N^{2}\right) \int_{\partial F_{\varepsilon}}\left(w_{\varepsilon} \times n_{\varepsilon}\right) \cdot R_{\varepsilon} v d \sigma+\varepsilon^{-1} \int_{\Omega_{\varepsilon}} f \cdot R_{\varepsilon} v d x .
\end{aligned}
$$

Applying Cauchy-Schwarz's inequality and taking into account estimates of the velocity (4.45), microrotation (4.46) and restricted operator (4.49), we get

$$
\begin{aligned}
\left|\int_{\Omega_{\varepsilon}} \operatorname{rot}\left(u_{\varepsilon}\right) \cdot \operatorname{rot}\left(R_{\varepsilon} v\right) d x\right| & \leq C\left\|D u_{\varepsilon}\right\|_{L^{2}\left(\Omega_{\varepsilon}\right)^{3 \times 3}}\left\|D R_{\varepsilon} v\right\|_{L^{2}\left(\Omega_{\varepsilon}\right)^{3 \times 3}} \leq C \varepsilon^{-1}\|v\|_{H_{0}^{1}(\Omega)}, \\
\left|\int_{\Omega_{\varepsilon}} \operatorname{rot}\left(R_{\varepsilon} v\right) \cdot w_{\varepsilon} d x\right| & \leq C\left\|D R_{\varepsilon} v\right\|_{L^{2}\left(\Omega_{\varepsilon}\right)^{3 \times 3}}\left\|w_{\varepsilon}\right\|_{L^{2}\left(\Omega_{\varepsilon}\right)^{3}} \leq C \varepsilon^{-1}\|v\|_{H_{0}^{1}(\Omega)}, \\
\left|\varepsilon^{-1} \int_{\Omega_{\varepsilon}} f \cdot R_{\varepsilon} v d x\right| & \leq \varepsilon^{-1}\|f\|_{L^{2}(\Omega)^{3}}\left\|R_{\varepsilon} v\right\|_{L^{2}\left(\Omega_{\varepsilon}\right)^{3}} \leq C \varepsilon^{-1}\|v\|_{H_{0}^{1}(\Omega)} .
\end{aligned}
$$

From estimate (4.30) applied to $R_{\varepsilon} v$ and using estimate (4.49), we deduce $\left\|R_{\varepsilon} v\right\|_{L^{2}\left(\partial F_{\varepsilon}\right)^{3}} \leq C \varepsilon^{-\frac{1}{2}}$ and from estimate (4.31) with estimate (4.46), we deduce $\|w\|_{L^{2}\left(\partial F_{\varepsilon}\right)^{3}} \leq C \varepsilon^{-\frac{1}{2}}$. Then, we get

$$
\left|\int_{\partial F_{\varepsilon}}\left(w_{\varepsilon} \times n_{\varepsilon}\right) \cdot R_{\varepsilon} v d \sigma\right| \leq C\left\|w_{\varepsilon}\right\|_{L^{2}\left(\partial F_{\varepsilon}\right)^{3}}\left\|R_{\varepsilon} v\right\|_{L^{2}\left(\partial F_{\varepsilon}\right)^{3}} \leq C \varepsilon^{-\frac{1}{2}}\left\|R_{\varepsilon} v\right\|_{L^{2}\left(\partial F_{\varepsilon}\right)^{3}} \leq C \varepsilon^{-1}\|v\|_{H_{0}^{1}(\Omega)} .
$$

This together with previous inequalities gives $\left|\left\langle\nabla P_{\varepsilon}, v\right\rangle\right| \leq C \varepsilon^{-1}\|v\|_{H_{0}^{1}(\Omega)^{3}}$ and so $\left\|\nabla P_{\varepsilon}\right\|_{H^{-1}(\Omega)^{3}} \leq C \varepsilon^{-1}$. By using the classical inequality (see [33])

$$
\left\|P_{\varepsilon}\right\|_{L^{2}(\Omega)} \leq C\left\|\nabla P_{\varepsilon}\right\|_{H^{-1}(\Omega)^{3}},
$$

we get estimate (4.51).

A compactness result. Our aim is to describe the asymptotic behavior of the velocity $u_{\varepsilon}$, microrotation $w_{\varepsilon}$ and pressure $p_{\varepsilon}$ of the fluid as $\varepsilon$ tends to 0 taking into account the boundary of the obstacles. To do this we use the periodic unfolding method in perforated domains and the estimates given in the previous section. Thus, we briefly recall the definition of the unfolding operator and its main properties (for more details, see [15, 22] for fixed domains and $[17,18,19]$ for perforated domains).

In the sequel we will use the following notation:

- $\tilde{\varphi}$ for the zero extension outside $\Omega_{\varepsilon}$ (resp. $\Omega$ ) for any function $\varphi$ in $L^{2}\left(\Omega_{\varepsilon}\right)$ (resp. $L^{2}(\Omega)$ ),

- For $x \in \mathbb{R}^{3}$, we set $x=[x]_{Y}+\{x\}_{Y}$ where the integer part $[x]_{Y}$ belongs to the periodical net of $\mathbb{R}^{3}$ (i.e. the subgroup $\mathbb{Z}^{3}$ ) with respect to $Y$ and $\{x\}_{Y}=x-[x]_{Y}$ is the fractional part of $x$. Thus, for every $\varepsilon>0$, the former decomposition implies that we also have $x=\varepsilon\{x / \varepsilon\}_{Y}+\varepsilon[x / \varepsilon]_{Y}$ for every $x \in \mathbb{R}^{3}$. 
Definition 4.11. The unfolding operator $\mathcal{T}_{\varepsilon}: L^{2}\left(\Omega_{\varepsilon}\right) \rightarrow L^{2}\left(\mathbb{R}^{3} \times Y^{*}\right)$ is defined by

$$
\mathcal{T}_{\varepsilon}(\varphi)(x, y)=\tilde{\varphi}\left(\varepsilon\left[\frac{x}{\varepsilon}\right]_{Y}+\varepsilon y\right), \quad \forall \varphi \in L^{2}\left(\Omega_{\varepsilon}\right), \quad \forall(x, y) \in \mathbb{R}^{3} \times Y^{*} .
$$

Proposition 4.12. The unfolding operator $\mathcal{T}_{\varepsilon}$ has the following properties:

1. $\mathcal{T}_{\varepsilon}$ is a linear operator.

2. $\mathcal{T}_{\varepsilon}(\varphi \phi)=\mathcal{T}_{\varepsilon}(\varphi) \mathcal{T}_{\varepsilon}(\phi), \forall \varphi, \phi \in L^{2}\left(\Omega_{\varepsilon}\right)$.

3. $\mathcal{T}_{\varepsilon}\left(\varphi_{\varepsilon}\right)(x, y)=\varphi(y), \forall(x, y) \in \mathbb{R}^{3} \times Y^{*}, \forall \varphi \in L^{2}\left(Y^{*}\right)$ a $Y$-periodic function with $\varphi_{\varepsilon}(x)=\varphi\left(\frac{x}{\varepsilon}\right)$.

4. $\left\|\mathcal{T}_{\varepsilon}(\varphi)\right\|_{L^{2}\left(\mathbb{R}^{3} \times Y^{*}\right)}=|Y|^{\frac{1}{2}}\|\varphi\|_{L^{2}\left(\Omega_{\varepsilon}\right)}, \forall \varphi \in L^{2}\left(\Omega_{\varepsilon}\right)$.

5. $\nabla_{y} \mathcal{T}_{\varepsilon}(\varphi)(x, y)=\varepsilon \mathcal{T}_{\varepsilon}\left(\nabla_{x} \varphi\right)(x, y), \forall(x, y) \in \mathbb{R}^{3} \times Y^{*}, \forall \varphi \in H^{1}\left(\Omega_{\varepsilon}\right)$.

6. $\mathcal{T}_{\varepsilon}(\varphi) \in L^{2}\left(\mathbb{R}^{3} ; H^{1}\left(Y^{*}\right)\right), \forall \varphi \in H^{1}\left(\Omega_{\varepsilon}\right)$.

7. Let $\varphi_{\varepsilon}$ be in $L^{2}(\Omega)$ such that $\tilde{\varphi}_{\varepsilon} \rightarrow \varphi$ in $L^{2}(\Omega)$. Then $\mathcal{T}_{\varepsilon}\left(\varphi_{\varepsilon}\right) \rightarrow \tilde{\varphi}$ in $L^{2}\left(\mathbb{R}^{3} \times Y^{*}\right)$.

Proposition 4.13. Let $\varphi_{\varepsilon}$ be a sequence such that

$$
\left\|\varphi_{\varepsilon}\right\|_{L^{2}\left(\Omega_{\varepsilon}\right)^{3}}+\varepsilon\left\|D \varphi_{\varepsilon}\right\|_{L^{2}\left(\Omega_{\varepsilon}\right)^{3 \times 3}} \leq C .
$$

Then, there exists $\hat{\varphi} \in L^{2}\left(\Omega ; H_{\mathrm{per}}^{1}\left(Y^{*}\right)^{3}\right)$ such that

$$
\mathcal{T}_{\varepsilon}\left(\varphi_{\varepsilon}\right) \rightarrow \hat{\varphi} \quad \text { in } L^{2}\left(\Omega ; H^{1}\left(Y^{*}\right)^{3}\right), \quad \varepsilon \mathcal{T}_{\varepsilon}\left(D \varphi_{\varepsilon}\right) \rightarrow D_{y} \hat{\varphi} \quad \text { in } L^{2}\left(\Omega \times Y^{*}\right)^{3 \times 3} .
$$

In a similar way, it is introduced in $[17,19]$ the unfolding operator on the boundary of the holes $\partial F_{\varepsilon}$.

Definition 4.14. The unfolding boundary operator $\mathcal{T}_{\varepsilon}^{b}(\varphi) \in L^{2}\left(\mathbb{R}^{3} \times \partial F\right)$ is defined by

$$
\mathcal{T}_{\varepsilon}^{b}(\varphi)(x, y)=\tilde{\varphi}\left(\varepsilon\left[\frac{x}{\varepsilon}\right]_{Y}+\varepsilon y\right), \quad \forall \varphi \in L^{2}\left(\partial F_{\varepsilon}\right), \quad \forall(x, y) \in \mathbb{R}^{3} \times \partial F .
$$

We remark that if $\varphi \in H^{1}\left(\Omega_{\varepsilon}\right)$ and $\varphi=0$ on $\partial \Omega$, one has $\mathcal{T}_{\varepsilon}^{b}(\varphi)=\mathcal{T}_{\varepsilon}(\varphi)$ on $\partial F$. The next results reformulate the properties given above in the case of functions defined on the boundary of the holes $\partial F_{\varepsilon}$.

Proposition 4.15. The unfolding boundary operator $\mathcal{T}_{\varepsilon}^{b}$ has the following properties:

1. $\mathcal{T}_{\varepsilon}^{b}$ is linear.

2. $\mathcal{T}_{\varepsilon}^{b}(\varphi \phi)=\mathcal{T}_{\varepsilon}^{b}(\varphi) \mathcal{T}_{\varepsilon}^{b}(\phi), \forall \varphi, \phi \in L^{2}\left(\partial F_{\varepsilon}\right)$.

3. $\mathcal{T}_{\varepsilon}^{b}\left(\varphi_{\varepsilon}\right)(x, y)=\varphi(y), \quad \forall(x, y) \in \mathbb{R}^{3} \times \partial F, \forall \varphi \in L^{2}(\partial F)$ a $Y$-periodic function with $\varphi_{\varepsilon}(x)=\varphi\left(\frac{x}{\varepsilon}\right)$.

4. $\left\|\mathcal{T}_{\varepsilon}^{b}(\varphi)\right\|_{L^{p}\left(\mathbb{R}^{3} \times \partial F\right)}=(\varepsilon|Y|)^{\frac{1}{2}}\|\varphi\|_{L^{p}\left(\partial F_{\varepsilon}\right)}, \forall \varphi \in L^{2}\left(\partial F_{\varepsilon}\right)$.

5. $\lim _{\varepsilon \rightarrow 0} \int_{\mathbb{R}^{3} \times \partial F} \mathcal{T}_{\varepsilon}^{b}(\varphi)(x, y) d x d \sigma(y)=|\partial F| \int_{\Omega} \varphi(x) d x, \forall \varphi \in H^{1}(\Omega)$.

6. $\mathcal{T}_{\varepsilon}^{b}(\varphi) \rightarrow \tilde{\varphi}$ strongly in $L^{2}\left(\mathbb{R}^{3} \times \partial F\right), \forall \varphi \in H_{0}^{1}(\Omega)$.

Next, we give some compactness results about the behavior of the extended functions $\left(U_{\varepsilon}, W_{\varepsilon}, P_{\varepsilon}\right)$ and the unfolding functions $\left(\mathcal{T}_{\varepsilon}\left(u_{\varepsilon}\right), \mathcal{T}_{\varepsilon}\left(w_{\varepsilon}\right), \mathcal{T}_{\varepsilon}\left(P_{\varepsilon}\right)\right)$ by assuming the a priori estimates given in Lemmas 4.5, 4.7 and 4.10.

Proposition 4.16. Assume that the asymptotic regimes (2.14) and (2.18) and condition (3.26) hold. Then, for a subsequence of $\varepsilon$ still denoted by $\varepsilon$, we have that 
1. (Velocity) there exist $u \in L^{2}(\Omega)^{3}$ and $\hat{u} \in L^{2}\left(\Omega ; H_{\mathrm{per}}^{1}\left(Y^{*}\right)^{3}\right)$ such that

$$
\begin{gathered}
\varepsilon^{-1} U_{\varepsilon} \rightarrow u \text { in } L^{2}(\Omega)^{3}, \\
\varepsilon^{-1} \mathcal{T}_{\varepsilon}\left(u_{\varepsilon}\right) \rightarrow \hat{u} \text { in } L^{2}\left(\Omega ; H^{1}\left(Y^{*}\right)\right)^{3}, \quad \mathcal{T}_{\varepsilon}\left(D u_{\varepsilon}\right) \rightarrow D_{y} \hat{u} \text { in } L^{2}\left(\Omega \times Y^{*}\right)^{3 \times 3}, \\
\varepsilon^{-1} \mathcal{T}_{\varepsilon}^{b}\left(u_{\varepsilon}\right) \rightarrow \hat{u} \text { in } L^{2}\left(\Omega ; H^{\frac{1}{2}}(\partial F)\right)^{3}, \\
\mathcal{T}_{\varepsilon}\left(\operatorname{rot}\left(u_{\varepsilon}\right)\right) \rightarrow \operatorname{rot}_{y}(\hat{u}) \text { in } L^{2}\left(\Omega \times Y^{*}\right)^{3},
\end{gathered}
$$

and moreover, the following conditions hold

$$
\begin{aligned}
u(x)=\int_{Y^{*}} \hat{u}(x, y) d y & \text { in } \Omega, \\
\hat{u}(x, y) \cdot n(y)=0 & \text { on } \Omega \times \partial F, \\
\operatorname{div}_{y} \hat{u}(x, y)=0 & \text { in } \Omega \times Y^{*}, \\
\operatorname{div}_{x}\left(\int_{Y^{*}} \hat{u}(x, y) d y\right)=0 & \text { in } \Omega, \\
\left(\int_{Y^{*}} \hat{u}(x, y) d y\right) \cdot n(y)=0 & \text { in } \Omega,
\end{aligned}
$$

2. (Microrotation) there exist $w \in L^{2}(\Omega)^{3}$ and $\hat{w} \in L^{2}\left(\Omega ; H_{\mathrm{per}}^{1}\left(Y^{*}\right)^{3}\right)$ such that

$$
\begin{gathered}
W_{\varepsilon} \rightarrow w \text { in } L^{2}(\Omega)^{3}, \\
\mathcal{T}_{\varepsilon}\left(w_{\varepsilon}\right) \rightarrow \hat{w} \text { in } L^{2}\left(\Omega ; H^{1}\left(Y^{*}\right)\right)^{3}, \quad \varepsilon \mathcal{T}_{\varepsilon}\left(D w_{\varepsilon}\right) \rightarrow D_{y} \hat{w} \text { in } L^{2}\left(\Omega \times Y^{*}\right)^{3 \times 3}, \\
\mathcal{T}_{\varepsilon}^{b}\left(w_{\varepsilon}\right) \rightarrow \hat{w} \text { in } L^{2}\left(\Omega ; H^{\frac{1}{2}}(\partial F)\right)^{3}, \\
\varepsilon \mathcal{T}_{\varepsilon}\left(\operatorname{rot}\left(w_{\varepsilon}\right)\right) \rightarrow \operatorname{rot}_{y}(\hat{u}) \text { in } L^{2}\left(\Omega \times Y^{*}\right)^{3},
\end{gathered}
$$

and moreover, the following conditions hold

$$
\begin{aligned}
w(x)=\int_{Y^{*}} \hat{w}(x, y) d y & \text { in } \Omega, \\
\hat{w}(x, y) \cdot n(y)=0 & \text { on } \Omega \times \partial F,
\end{aligned}
$$

3. (Pressure) there exist $p \in L_{0}^{2}(\Omega)^{3}$ such that

$$
\varepsilon P_{\varepsilon} \rightarrow p \text { in } L^{2}(\Omega), \quad \varepsilon \mathcal{T}_{\varepsilon}\left(P_{\varepsilon}\right) \rightarrow p \text { in } L^{2}\left(\Omega \times Y^{*}\right) .
$$

Proof. We start proving 1. From estimates for the extended velocity (4.47) we deduce convergence (4.54). Taking into account the a priori estimates for the velocity (4.45) and using Proposition 4.13, we deduce convergences given in the (4.55). Convergence (4.56) is straightforward from the definition (4.53) and the Sobolev injections. Finally, taking into account Proposition $4.12_{1,5}$, we have

$$
\mathcal{T}_{\varepsilon}\left(\partial_{x_{i}} u_{\varepsilon, j}-\partial_{x_{j}} u_{\varepsilon, i}\right)=\mathcal{T}_{\varepsilon}\left(\partial_{x_{i}} u_{\varepsilon, j}\right)-\mathcal{T}_{\varepsilon}\left(\partial_{x_{j}} u_{\varepsilon, i}\right)=\varepsilon^{-1}\left(\partial_{y_{i}} \mathcal{T}_{\varepsilon}\left(u_{\varepsilon, j}\right)-\partial_{y_{j}} \mathcal{T}_{\varepsilon}\left(u_{\varepsilon, i}\right)\right), \quad \forall i, j=1,2,3, i<j,
$$

and so $\mathcal{T}_{\varepsilon}\left(\operatorname{rot}\left(u_{\varepsilon}\right)\right)=\varepsilon^{-1} \operatorname{rot}_{y}\left(\mathcal{T}_{\varepsilon}\left(u_{\varepsilon}\right)\right)$, which from convergence (4.55) implies (4.57).

In order to prove the boundary conditions (4.59), let us take $\varphi \in \mathcal{D}(\Omega)$ and from $u_{\varepsilon} \cdot n_{\varepsilon}=0$ on $\partial F_{\varepsilon}$, we have

$$
\int_{\partial F_{\varepsilon}}\left(u_{\varepsilon} \cdot n_{\varepsilon}\right) \varphi d \sigma(x)=0
$$


By applying the unfolding boundary and using Proposition $4.15_{2,3,4}$, we get

$$
0=\int_{\partial F_{\varepsilon}}\left(u_{\varepsilon} \cdot n_{\varepsilon}\right) \varphi d \sigma(x)=\varepsilon^{-1} \int_{\mathbb{R}^{3} \times \partial F}\left(\mathcal{T}_{\varepsilon}^{b}\left(u_{\varepsilon}\right) \cdot n\right) \mathcal{T}_{\varepsilon}^{b}(\varphi) d x d \sigma(y) .
$$

Passing to the limit when $\varepsilon$ tends to zero, from convergence (4.56) and Proposition $4.15_{6}$, we obtain

$$
0=\int_{\Omega \times \partial F}(\hat{u}(x, y) \cdot n(y)) \varphi d x d \sigma(y)=\int_{\Omega}\left(\int_{\partial F} \hat{u}(x, y) \cdot n(y) d y\right) \varphi(x) d x,
$$

which implies (4.59).

In order to prove relation (4.60), let us observe that $\operatorname{div}\left(u_{\varepsilon}\right)=0 \operatorname{implies} \mathcal{T}_{\varepsilon}\left(\operatorname{div}\left(u_{\varepsilon}\right)\right)=0$. But from Proposition $4.12_{1,5}$, we have

$$
\mathcal{T}_{\varepsilon}\left(\operatorname{div}\left(u_{\varepsilon}\right)\right)=\sum_{i=1}^{3} \mathcal{T}_{\varepsilon}\left(\partial_{x_{i}} u_{\varepsilon, i}\right)=\varepsilon^{-1} \sum_{i=1}^{3} \partial_{y_{i}} \mathcal{T}_{\varepsilon}\left(u_{\varepsilon, i}\right)=\varepsilon^{-1} \operatorname{div}_{y}\left(\mathcal{T}_{\varepsilon}\left(u_{\varepsilon}\right)\right)
$$

and so $\varepsilon^{-1} \operatorname{div}_{y}\left(\mathcal{T}_{\varepsilon}\left(u_{\varepsilon}\right)\right)=0$. Passing to the limit as $\varepsilon$ tends to zero in the last equality we get (4.60).

In order to prove (4.59) and (4.61), multiplying $\operatorname{div}\left(u_{\varepsilon}\right)=0$ by $\varepsilon^{-1} \varphi$ with $\varphi$ in $\mathcal{D}(\Omega)$ and using $u_{\varepsilon} \cdot n_{\varepsilon}=0$ on $\partial F_{\varepsilon}$, we have

$$
0=\int_{\Omega_{\varepsilon}} \varepsilon^{-1} \operatorname{div}\left(u_{\varepsilon}\right) \varphi d x=\int_{\Omega_{\varepsilon}} \varepsilon^{-1} u_{\varepsilon} \cdot \nabla \varphi d x
$$

By applying the unfolding, we get

$$
\int_{\mathbb{R}^{3} \times Y^{*}} \varepsilon^{-1} \mathcal{T}_{\varepsilon}\left(u_{\varepsilon}\right) \cdot \mathcal{T}_{\varepsilon}(\nabla \varphi) d x d y=0
$$

We pass to the limit as $\varepsilon$ tends to zero and we get

$$
\int_{\Omega \times Y^{*}} \hat{u}(x, y) \cdot \nabla \varphi(x) d x d y=0
$$

and so

$$
\int_{\Omega} \operatorname{div}_{x}\left(\int_{Y^{*}} \hat{u}(x, y) d y\right) \varphi(x) d x=0, \quad \forall \varphi \in \mathcal{D}(\Omega)
$$

which implies (4.61) and (4.62).

Finally, we prove (4.58). From Proposition 4.12 and taking $\varphi_{\varepsilon}=\varepsilon^{-1} \varphi$ with $\varphi$ in $\mathcal{D}(\Omega)$, we have

$$
\varepsilon^{-1} \int_{\Omega_{\varepsilon}} u_{\varepsilon}(x) \cdot \varphi(x) d x=\frac{1}{\varepsilon|Y|} \int_{\mathbb{R}^{3} \times Y^{*}} \mathcal{T}_{\varepsilon}\left(u_{\varepsilon}\right)(x, y) \cdot \mathcal{T}_{\varepsilon}(\varphi)(x, y) d x d y .
$$

By using the extension of the velocity, we have

$$
\varepsilon^{-1} \int_{\Omega} U_{\varepsilon}(x) \cdot \varphi(x) d x=\varepsilon^{-1} \int_{\mathbb{R}^{3} \times Y^{*}} \mathcal{T}_{\varepsilon}\left(u_{\varepsilon}\right)(x, y) \cdot \mathcal{T}_{\varepsilon}(\varphi)(x, y) d x d y,
$$

and passing to the limit by using convergences (4.54) and (4.55), we get

$$
\int_{\Omega} u(x) \cdot \varphi(x) d x=\int_{\Omega}\left(\int_{Y^{*}} \hat{u}(x, y) d y\right) \varphi(x) d x,
$$

which implies property (4.58).

The proof of 2 is similar, so we omit it.

We finish with the proof of 3. Thus, estimates given in Lemma 4.10 imply, up to a subsequence, the existence of $p \in L_{0}^{2}(\Omega)$ such that

$$
\varepsilon P_{\varepsilon} \rightarrow p \text { in } L^{2}(\Omega), \quad \varepsilon \nabla P_{\varepsilon} \rightarrow p \text { in } H^{-1}(\Omega)^{3} .
$$


Moreover, following [32] it can be proved that this convergence is in fact strong. To prove this, let $\sigma_{\varepsilon} \in H_{0}^{1}(\Omega)^{3}$ such that

$$
\sigma_{\varepsilon} \rightarrow \sigma \text { in } H_{0}^{1}(\Omega)^{3}
$$

Then, we have

$$
\left|\left\langle\varepsilon \nabla P_{\varepsilon}, \sigma_{\varepsilon}\right\rangle_{H^{-1}, H_{0}^{1}(\Omega)^{3}}\right| \leq\left|\left\langle\varepsilon \nabla P_{\varepsilon}, \sigma_{\varepsilon}-\sigma\right\rangle_{H^{-1}, H_{0}^{1}(\Omega)^{3}}\right|+\left|\left\langle\varepsilon\left(\nabla P_{\varepsilon}-\nabla p\right), \sigma\right\rangle_{H^{-1}, H_{0}^{1}(\Omega)^{3}}\right| .
$$

On the one hand, using first convergence in (4.70), we have

$$
\left|\left\langle\varepsilon\left(\nabla P_{\varepsilon}-\nabla p\right), \sigma\right\rangle_{H^{-1}, H_{0}^{1}(\Omega)^{3}}\right|=\left|\int_{\Omega} \varepsilon\left(P_{\varepsilon}-p\right) \operatorname{div}(\sigma) d x\right| \rightarrow 0, \quad \text { as } \varepsilon \rightarrow 0 .
$$

On the other hand, from estimate (4.49) and (4.51), we have that

$$
\begin{aligned}
\left|\left\langle\varepsilon \nabla P_{\varepsilon}, \sigma_{\varepsilon}-\sigma\right\rangle_{H^{-1}, H_{0}^{1}(\Omega)^{3}}\right| & =\left|\left\langle\varepsilon \nabla P_{\varepsilon}, R_{\varepsilon}\left(\sigma_{\varepsilon}-\sigma\right)\right\rangle_{H^{-1}, H_{0}^{1}\left(\Omega_{\varepsilon}\right)^{3}}\right| \\
& \leq C\left(\left\|\sigma_{\varepsilon}-\sigma\right\|_{L^{2}(\Omega)^{3}}+\varepsilon\left\|D\left(\sigma_{\varepsilon}-\sigma\right)\right\|_{L^{2}(\Omega)^{3}}\right) \rightarrow 0, \text { as } \varepsilon \rightarrow 0,
\end{aligned}
$$

by virtue of (4.71) and the Rellich theorem. This implies that $\nabla P_{\varepsilon} \rightarrow \nabla p$ in $H^{-1}(\Omega)^{3}$, which together with inequality (4.52), implies the strong convergence of the pressure $P_{\varepsilon}$ given in (4.69). This convergence and Proposition $4.12_{7}$ imply the strong convergence of $\varepsilon \mathcal{T}_{\varepsilon}\left(P_{\varepsilon}\right)$ to $p$ in $L^{2}\left(\Omega \times Y^{*}\right)$.

Obtaining the limit system. We use the results of the previous sections to prove Theorem 3.3 describing the asymptotic behavior of the solution of the micropolar system (2.15)-(2.23). To do this, we first give the existence and uniqueness result for micropolar local problem (3.29).

Lemma 4.17. Assume that condition (3.26) holds. Then, for every $k=1,2$ and $i=1,2,3$, there exists a unique solution $\left(u^{i, k}, w^{i, k}, \pi^{i, k}\right) \in V_{Y}^{0} \times V_{Y} \times L_{0}^{2}\left(Y^{*}\right)$ of the local problem (3.29).

Proof. Similarly to Proposition 3.1, sufficiently regular solutions of (3.29) satisfy the following weak formulation: For $i=1,2,3, k=1,2$ find $\left(u^{i, k}, w^{i, k}, \pi^{i, k}\right) \in V_{Y}^{0} \times V_{Y} \times L_{0}^{2}\left(Y^{*}\right)$ such that

$$
\begin{gathered}
\int_{Y^{*}} \operatorname{rot}\left(u^{i, k}\right) \cdot \operatorname{rot}(\varphi) d x-\int_{Y^{*}} \pi^{i, k} \operatorname{div}(\varphi) d x-2 N^{2} \int_{Y^{*}} \operatorname{rot}(\varphi) \cdot w^{i, k} d x \\
+2\left(\frac{1}{\alpha}-N^{2}\right) \int_{\partial F}\left(w^{i, k} \times n\right) \cdot \varphi d \sigma=\int_{Y^{*}} e_{i} \delta_{1 k} \cdot \varphi d x, \forall \varphi \in V_{Y}, \\
R_{c} \int_{Y^{*}} \operatorname{rot}\left(w^{i, k}\right) \cdot \operatorname{rot}(\psi) d x+R_{c} \int_{Y^{*}} \operatorname{div}\left(w^{i, k}\right) \cdot \operatorname{div}(\psi) d x+4 N^{2} \int_{Y^{*}} w^{i, k} \cdot \psi d x \\
-2 N^{2} \int_{Y^{*}} \operatorname{rot}(\psi) \cdot u^{i, k} d x+2 N^{2}(\beta-1) \int_{\partial F}\left(u^{i, k} \times n\right) \cdot \psi d \sigma=\int_{Y^{*}} e_{i} \delta_{2 k} \cdot \psi d x, \quad \forall \psi \in V_{Y} .
\end{gathered}
$$

Thus, following the lines of the proof of Theorem 3.2, we can introduce the following mixed variational form:

For $i=1,2,3, k=1,2$ find $\left(u^{i, k}, w^{i, k}, \pi^{i, k}\right) \in V_{Y}^{0} \times V_{Y} \times L_{0, \text { per }}^{2}\left(Y^{*}\right)$ such that

$$
\begin{aligned}
\mathcal{A}_{Y}\left(u^{i, k}, w^{i, k} ; \varphi, \psi\right)+\mathcal{B}_{Y}\left((\varphi, \psi), \pi^{i, k}\right) & =\mathcal{L}_{Y}^{i, k}(\varphi, \psi) & & \forall(\varphi, \psi) \in V_{Y} \times V, \\
\mathcal{B}_{\varepsilon}\left(\left(u^{i, k}, w^{i, k}\right), q^{i, k}\right) & =0 & & \forall q^{i, k} \in L_{0, \mathrm{per}}^{2}\left(Y^{*}\right),
\end{aligned}
$$


where

$$
\begin{aligned}
\mathcal{A}_{Y}\left(u^{i, k}, w^{i, k} ; \varphi, \psi\right)= & \int_{Y^{*}} \operatorname{rot}_{y}\left(u^{i, k}\right) \cdot \operatorname{rot}_{y}(\varphi) d x-2 N^{2} \int_{Y^{*}} \operatorname{rot}_{y}(\varphi) \cdot w^{i, k} d y \\
& +R_{c} \int_{Y^{*}} \operatorname{rot}_{y}\left(w^{i, k}\right) \cdot \operatorname{rot}_{y}(\psi) d y+R_{c} \int_{Y^{*}} \operatorname{div}_{y}\left(w^{i, k}\right) \cdot \operatorname{div}_{y}(\psi) d y \\
& -2 N^{2} \int_{Y^{*}} \operatorname{rot}_{y}(\psi) \cdot u^{i, k} d y+4 N^{2} \int_{Y^{*}} w^{i, k} \cdot \psi d y \\
& +2\left(\frac{1}{\alpha}-N^{2}\right) \int_{\partial F}\left(w^{i, k} \times n\right) \cdot \varphi d \sigma(y)+2 N^{2}(\beta-1) \int_{\partial F}\left(u^{i, k} \times n\right) \cdot \psi d \sigma(y),
\end{aligned}
$$

and

We denote

$$
\mathcal{B}_{Y}\left((\varphi, \psi), \pi^{i, k}\right)=-\int_{Y^{*}} \pi^{i, k} \operatorname{div}_{y} \varphi d y, \quad \mathcal{L}_{Y}^{i, k}(\varphi, \psi)=\int_{Y^{*}} e_{i} \delta_{1 k} \cdot \varphi d y+\int_{Y^{*}} e_{i} \delta_{2 k} \cdot \psi d y .
$$

$$
\|(\varphi, \psi)\|_{V_{Y}^{0} \times V_{Y}}=\left(\|D \varphi\|_{L^{2}\left(Y^{*}\right)^{3 \times 3}}^{2}+\|D \psi\|_{L^{2}\left(Y^{*}\right)^{3 \times 3}}^{2}\right)^{\frac{1}{2}} .
$$

Following the proof of Theorem 3.2 and taking into account that in $Y^{*}$ the trace inequality (4.30) holds with constant $C_{t}$ instead of $\varepsilon^{-1} C_{t}$, the Poincaré inequality (4.31) with constant $C_{p}$ instead of $\varepsilon C_{p}$, the trace inequality (4.33) with constant $C_{p t}^{\frac{1}{2}}$ instead of $\left(\varepsilon C_{p t}\right)^{\frac{1}{2}}$ and the Gaffney inequality (4.34) with constant $C_{g}$, it is not difficult to prove that the bilinear form $\mathcal{A}_{Y}, \mathcal{B}_{Y}$ and $\mathcal{L}_{Y}$ are continuous bilinear forms on $\left(V_{Y}^{0} \times V_{Y}\right)^{2}, V_{Y} \times V_{Y} \times L_{0, \text { per }}^{2}\left(Y^{*}\right)$ and $V_{Y} \times V_{Y}$ respectively. . Moreover, under condition (3.26) it follows from the proof of the coercivity of $\mathcal{A}_{\varepsilon}$ in Theorem 3.2 that $\mathcal{A}_{Y}$ satisfies

$$
\mathcal{A}_{Y}(\varphi, \psi ; \varphi, \psi) \geq A\|D \varphi\|_{L^{2}\left(\Omega_{\varepsilon}\right)^{3 \times 3}}^{2}+B\|D \psi\|_{L^{2}\left(\Omega_{\varepsilon}\right)^{3 \times 3}}^{2} \geq \min \{A, B\}\|(\varphi, \psi)\|_{V_{Y}^{0} \times V_{Y}}^{2},
$$

with positive constants $A, B$ given by (4.42). Moreover, it can be proved the inf-sup condition

$$
\exists \delta>0, \quad \text { such that } \sup _{(\varphi, \psi) \in V_{Y} \times V_{Y}} \frac{\int_{Y^{*}} \operatorname{div} \varphi q^{i, k} d x}{\left(\|\varphi\|_{V_{Y}}^{2}+\|\varphi\|_{V_{Y}}^{2}\right)^{\frac{1}{2}}} \geq \delta,
$$

which ends the proof.

Proof of Theorem 3.3. We divide the proof in two steps.

Step 1. In this step we prove that the whole sequences $\left(\varepsilon^{-1} \mathcal{T}_{\varepsilon}\left(u_{\varepsilon}\right), \mathcal{T}_{\varepsilon}\left(w_{\varepsilon}\right)\right)$ and $\varepsilon \mathcal{T}_{\varepsilon}\left(P_{\varepsilon}\right)$ converge weakly in $L^{2}\left(\Omega ; H^{1}\left(Y^{*}\right)^{3}\right) \times L^{2}\left(\Omega ; H^{1}\left(Y^{*}\right)^{3}\right)$ to $(\hat{u}, \hat{w})$ and strongly to $p$ in $L^{2}\left(\Omega \times Y^{*}\right)$ respectively, where the triplet $(\hat{u}, \hat{w}, p) \in L^{2}\left(\Omega ; H_{\mathrm{per}}^{1}\left(Y^{*}\right)^{3}\right) \times L^{2}\left(\Omega ; H_{\mathrm{per}}^{1}\left(Y^{*}\right)^{3}\right) \times L_{0}^{2}(\Omega)$ is the unique solution of the following homogenized system

$$
\begin{array}{rc}
-\Delta_{y} \hat{u}+\nabla_{y} \hat{q}-2 N^{2} \operatorname{rot}_{y}(\hat{w})=f-\nabla_{x} p & \text { in } \Omega \times Y^{*}, \\
-R_{c} \Delta_{y} \hat{w}+4 N^{2} \hat{w}-2 N^{2} \operatorname{rot}_{y}(\hat{u})=g & \text { in } \Omega \times Y^{*}, \\
\operatorname{div}_{y}(\hat{u})=0 & \text { in } \Omega \times Y^{*}, \\
\operatorname{div}_{x}\left(\int_{Y^{*}} \hat{u} d y\right)=0 & \text { in } \Omega, \\
\left(\int_{Y^{*}} \hat{u} d y\right) \cdot n=0 & \text { in } \Omega,
\end{array}
$$


where $\hat{q} \in L^{2}\left(\Omega ; L_{0 \text {,per }}^{2}\left(Y^{*}\right)^{3}\right)$, and the boundary conditions

$$
\begin{array}{rr}
\hat{w} \times n=\frac{\alpha}{2} \operatorname{rot}_{y}(\hat{u}) \times n & \text { on } \Omega \times \partial F, \\
\operatorname{rot}_{y}(\hat{w}) \times n=\frac{2 N^{2}}{R_{c}} \beta(\hat{u} \times n) & \text { on } \Omega \times \partial F, \\
\hat{u} \cdot n=0 & \text { on } \Omega \times \partial F, \\
\hat{w} \cdot n=0 & \text { on } \Omega \times \partial F .
\end{array}
$$

By taking into account Proposition 4.16, we have that (4.75)-(4.77) and (4.80)-(4.81) hold. Below, we prove the rest of them.

First, we prove (4.73). To do this, we first take as test function in (3.24) the following function $\varphi_{\varepsilon}(x)=$ $\varepsilon \phi(x) \Phi(x / \varepsilon)$, where $\phi \in \mathcal{D}(\Omega)$ and $\Phi \in H_{\text {per }}^{1}\left(Y^{*}\right)^{3}$ with $\Phi \cdot n=0$ on $\partial F$ and $\operatorname{div}_{y} \Phi=0$ in $Y^{*}$. Then, we have

$$
\begin{aligned}
& \int_{\Omega_{\varepsilon}} \operatorname{rot}\left(u_{\varepsilon}\right) \cdot \operatorname{rot}\left(\varphi_{\varepsilon}\right) d x-\int_{\Omega_{\varepsilon}} p_{\varepsilon} \operatorname{div}\left(\varphi_{\varepsilon}\right) d x-2 N^{2} \int_{\Omega_{\varepsilon}} \operatorname{rot}\left(\varphi_{\varepsilon}\right) \cdot w_{\varepsilon} d x \\
& +2\left(\frac{1}{\alpha}-N^{2}\right) \int_{\partial F_{\varepsilon}}\left(w_{\varepsilon} \times n_{\varepsilon}\right) \cdot \varphi_{\varepsilon} d \sigma(x)=\varepsilon^{-1} \int_{\Omega_{\varepsilon}} f \cdot \varphi_{\varepsilon} d x .
\end{aligned}
$$

Let us observe

$$
\operatorname{rot}\left(\varphi_{\varepsilon}\right)=\varepsilon \nabla \phi \times \Phi\left(\frac{\cdot}{\varepsilon}\right)+\phi \operatorname{rot}_{y}\left(\Phi\left(\frac{\cdot}{\varepsilon}\right)\right), \quad \operatorname{div}\left(\varphi_{\varepsilon}\right)=\varepsilon \nabla \phi \cdot \Phi\left(\frac{\cdot}{\varepsilon}\right)+\phi \operatorname{div}_{y}\left(\Phi\left(\frac{\cdot}{\varepsilon}\right)\right) .
$$

Hence, by Proposition $4.12_{2,3,7}$, we have $\mathcal{T}_{\varepsilon}\left(\varphi_{\varepsilon}\right)=\varepsilon \mathcal{T}_{\varepsilon}(\phi) \Phi$ and convergences

$$
\mathcal{T}_{\varepsilon}(\phi) \Phi \rightarrow \phi \Phi \text { in } L^{2}\left(\Omega \times Y^{*}\right)^{3}, \mathcal{T}_{\varepsilon}\left(\varphi_{\varepsilon}\right) \rightarrow 0 \text { in } L^{2}\left(\Omega \times Y^{*}\right)^{3}, \mathcal{T}_{\varepsilon}\left(\operatorname{rot}\left(\varphi_{\varepsilon}\right)\right) \rightarrow \phi \operatorname{rot}_{y}(\Phi) \text { in } L^{2}\left(\Omega \times Y^{*}\right)^{3} .
$$

By applying the unfolding and using Propositions $4.12_{2,3}$ and $4.15_{2,3}$, we get

$$
\begin{aligned}
& \int_{\mathbb{R}^{3} \times Y^{*}} \mathcal{T}_{\varepsilon}\left(\operatorname{rot}\left(u_{\varepsilon}\right)\right) \cdot \mathcal{T}_{\varepsilon}\left(\operatorname{rot}\left(\varphi_{\varepsilon}\right)\right) d x d y-\int_{\mathbb{R}^{3} \times Y^{*}} \mathcal{T}_{\varepsilon}\left(P_{\varepsilon}\right) \mathcal{T}_{\varepsilon}\left(\operatorname{div}\left(\varphi_{\varepsilon}\right)\right) d x d y-2 N^{2} \int_{\mathbb{R}^{3} \times Y^{*}} \mathcal{T}_{\varepsilon}\left(\operatorname{rot}\left(\varphi_{\varepsilon}\right)\right) \cdot \mathcal{T}_{\varepsilon}\left(w_{\varepsilon}\right) d x d y \\
& +2\left(\frac{1}{\alpha}-N^{2}\right) \int_{\mathbb{R}^{3} \times \partial F}\left(\mathcal{T}_{\varepsilon}^{b}\left(w_{\varepsilon}\right) \times n\right) \cdot\left(\mathcal{T}_{\varepsilon}^{b}(\phi) \Phi\right) d x d \sigma(y)=\int_{\mathbb{R}^{3} \times Y^{*}} \mathcal{T}_{\varepsilon}(f) \cdot\left(\mathcal{T}_{\varepsilon}(\phi) \Phi\right) d x d y .
\end{aligned}
$$

Next, we pass to the limit in every terms of the previous variational formulation:

- First term. From convergence (4.57) and (4.83), we have

$$
\begin{aligned}
\int_{\mathbb{R}^{3} \times Y^{*}} \mathcal{T}_{\varepsilon}\left(\operatorname{rot}\left(u_{\varepsilon}\right)\right) \cdot \mathcal{T}_{\varepsilon}\left(\operatorname{rot}\left(\varphi_{\varepsilon}\right)\right) d x d y & \rightarrow \int_{\Omega \times Y^{*}} \operatorname{rot}_{y}(\hat{u}(x, y)) \cdot\left(\phi(x) \operatorname{rot}_{y}(\Phi(y))\right) d x d y \\
& =\int_{\Omega \times Y^{*}} \operatorname{rot}_{y}(\hat{u}(x, y)) \cdot \operatorname{rot}_{y}(\phi(x) \Phi(y)) d x d y
\end{aligned}
$$

- Second term. We use (4.82) and Proposition $4.12_{1,2,7}$, the fact that $\operatorname{div}_{y}(\Phi)=0$ in $Y^{*}$ and convergence (4.69),

$$
\begin{aligned}
\int_{\mathbb{R}^{3} \times Y^{*}} \mathcal{T}_{\varepsilon}\left(P_{\varepsilon}\right) \mathcal{T}_{\varepsilon}\left(\operatorname{div}\left(\varphi_{\varepsilon}\right)\right) d x d y & =\int_{\mathbb{R}^{3} \times Y^{*}} \varepsilon \mathcal{T}_{\varepsilon}\left(P_{\varepsilon}\right) \mathcal{T}_{\varepsilon}(\nabla \phi \cdot \Phi) d x d y \\
& \rightarrow \int_{\Omega \times Y^{*}} p(x) \nabla \phi(x) \cdot \Phi(y) d x=\int_{\Omega \times Y^{*}} p(x) \operatorname{div}_{x}(\phi(x) \Phi(y)) d x d y .
\end{aligned}
$$


- Third term. From convergences (4.64) and (4.83), we get

$$
\begin{aligned}
\int_{\mathbb{R}^{3} \times Y^{*}} \mathcal{T}_{\varepsilon}\left(\operatorname{rot}\left(\varphi_{\varepsilon}\right)\right) \cdot \mathcal{T}_{\varepsilon}\left(w_{\varepsilon}\right) d x d y & \rightarrow \int_{\Omega \times Y^{*}} \phi(x) \operatorname{rot}_{y}(\Phi(y)) \cdot \hat{w}(x, y) d x d y \\
& =\int_{\Omega \times Y^{*}} \operatorname{rot}_{y}(\phi(x) \Phi(y)) \cdot \hat{w}(x, y) d x d y
\end{aligned}
$$

- Fourth term. We use convergence (4.65) and (4.83),

$$
\int_{\mathbb{R}^{3} \times \partial F}\left(\mathcal{T}_{\varepsilon}^{b}\left(w_{\varepsilon}\right) \times n\right) \cdot\left(\mathcal{T}_{\varepsilon}^{b}(\phi) \Phi\right) d x d \sigma(y) \rightarrow \int_{\Omega \times \partial F}(\hat{w}(x, y) \times n(y)) \cdot(\phi(x) \Phi(y)) d x d \sigma(y) .
$$

- Fifth term. From Proposition $4.12_{7}$ and convergence (4.83), we have

$$
\int_{\mathbb{R}^{3} \times Y^{*}} \mathcal{T}_{\varepsilon}(f) \cdot\left(\mathcal{T}_{\varepsilon}(\phi) \Phi\right) d x d y \rightarrow \int_{\Omega \times Y^{*}} f(x) \cdot(\phi(x) \Phi(y)) d x d y .
$$

Therefore, taking into account the previous convergences and denoting $\varphi(x, y)=\phi(x) \Phi(y)$, we obtain

$$
\begin{aligned}
& \int_{\Omega \times Y^{*}} \operatorname{rot}_{y}(\hat{u}(x, y)) \cdot \operatorname{rot}_{y}(\varphi(x, y)) d x d y-\int_{\Omega} p(x) \operatorname{div}_{x}(\varphi(x, y)) d x d y-2 N^{2} \int_{\Omega \times Y^{*}} \operatorname{rot}_{y}(\varphi(x, y)) \cdot \hat{w}(x, y) d x d y \\
& \quad+2\left(\frac{1}{\alpha}-N^{2}\right) \int_{\Omega \times \partial F}(\hat{w}(x, y) \times n(y)) \cdot \varphi(x, y) d x d \sigma(y)=\int_{\Omega \times Y^{*}} f(x) \cdot \varphi(x, y) d x d y .
\end{aligned}
$$

By density, this variational formulation holds for $\varphi \in \mathcal{V}$ with $\operatorname{div}_{y}(\varphi)=0$ in $\Omega \times Y^{*}$, where

$$
\mathcal{V}=\left\{\varphi \in L^{2}\left(\Omega ; H_{\mathrm{per}}^{1}\left(Y^{*}\right)^{3}\right): \varphi \cdot n=0 \text { on } \Omega \times \partial F\right\} .
$$

Then we easily find that the function $\hat{u}$ satisfies the variational formulation

$$
\begin{aligned}
& \int_{\Omega \times Y^{*}} \operatorname{rot}_{y}(\hat{u}(x, y)) \cdot \operatorname{rot}_{y}(\varphi(x, y)) d x d y-2 N^{2} \int_{\Omega \times Y^{*}} \operatorname{rot}_{y}(\varphi(x, y)) \cdot \hat{w}(x, y) d x d y \\
& \quad+2\left(\frac{1}{\alpha}-N^{2}\right) \int_{\Omega \times \partial F}(\hat{w}(x, y) \times n(y)) \cdot \varphi(x, y) d x d \sigma(y)=\int_{\Omega \times Y^{*}} f(x) \cdot \varphi(x, y) d x d y,
\end{aligned}
$$

for every $\varphi \in \mathcal{V}^{0}\left(Y^{*}\right)$ where

$$
\mathcal{V}^{0}\left(Y^{*}\right)=\left\{\begin{array}{l}
\varphi \in \mathcal{V}: \operatorname{div}_{y}(\varphi)=0 \text { in } \Omega \times Y^{*} \\
\operatorname{div}_{x}\left(\int_{Y^{*}} \varphi d y\right)=0 \text { in } \Omega, \quad\left(\int_{Y^{*}} \varphi d y\right) \cdot n=0 \text { on } \partial \Omega
\end{array}\right\} .
$$

Next, we prove (4.74). To do this, we take as test function in (3.24) the function $\psi_{\varepsilon}(x)=\eta(x) \Psi(x / \varepsilon)$, where $\eta \in \mathcal{D}(\Omega)$ and $\Psi \in H_{\text {per }}^{1}\left(Y^{*}\right)^{3}$ with $\Psi \cdot n=0$ on $\partial F$, and we have

$$
\begin{aligned}
& \varepsilon^{2} R_{c} \int_{\Omega_{\varepsilon}} \operatorname{rot}\left(w_{\varepsilon}\right) \cdot \operatorname{rot}\left(\psi_{\varepsilon}\right) d x+\varepsilon^{2} R_{c} \int_{\Omega_{\varepsilon}} \operatorname{div}\left(w_{\varepsilon}\right) \cdot \operatorname{div}\left(\psi_{\varepsilon}\right) d x+4 N^{2} \int_{\Omega_{\varepsilon}} w_{\varepsilon} \cdot \psi_{\varepsilon} d x \\
& \quad-2 N^{2} \int_{\Omega_{\varepsilon}} \operatorname{rot}\left(\psi_{\varepsilon}\right) \cdot u_{\varepsilon} d x+2 N^{2}(\beta-1) \int_{\partial F_{\varepsilon}}\left(u_{\varepsilon} \times n_{\varepsilon}\right) \cdot \psi_{\varepsilon} d \sigma=\int_{\Omega_{\varepsilon}} g \cdot \psi_{\varepsilon} d x
\end{aligned}
$$

Similarly to what happens with $\varphi_{\varepsilon}$ and taking into account that

$$
\operatorname{rot}\left(\psi_{\varepsilon}\right)=\nabla \eta \times \Psi\left(\frac{\dot{\varepsilon}}{\varepsilon}\right)+\varepsilon^{-1} \eta \operatorname{rot}_{y}\left(\Psi\left(\frac{\dot{\varepsilon}}{\varepsilon}\right)\right), \quad \operatorname{div}\left(\psi_{\varepsilon}\right)=\nabla \eta \cdot \Psi\left(\frac{\dot{\varepsilon}}{\varepsilon}\right)+\varepsilon^{-1} \eta \operatorname{div}_{y}\left(\Psi\left(\frac{\dot{\varepsilon}}{\varepsilon}\right)\right),
$$


we have $\mathcal{T}_{\varepsilon}\left(\psi_{\varepsilon}\right)=\mathcal{T}_{\varepsilon}(\eta) \Psi$ and

$$
\begin{gathered}
\mathcal{T}_{\varepsilon}\left(\psi_{\varepsilon}\right) \rightarrow \eta \Psi \quad \text { in } L^{2}\left(\Omega \times Y^{*}\right)^{3}, \\
\varepsilon \mathcal{T}_{\varepsilon}\left(\operatorname{div}\left(\psi_{\varepsilon}\right)\right) \rightarrow \eta \operatorname{div}_{y}(\Psi) \text { in } L^{2}\left(\Omega \times Y^{*}\right)^{3}, \quad \varepsilon \mathcal{T}_{\varepsilon}\left(\operatorname{rot}\left(\psi_{\varepsilon}\right)\right) \rightarrow \eta \operatorname{rot}_{y}(\Psi) \text { in } L^{2}\left(\Omega \times Y^{*}\right)^{3} .
\end{gathered}
$$

By applying the unfolding and using Propositions $4.12_{2,3}$ and $4.15_{2,3,4}$, we get

$$
\begin{aligned}
& \varepsilon^{2} R_{c} \int_{\mathbb{R}^{3} \times Y^{*}} \mathcal{T}_{\varepsilon}\left(\operatorname{rot}\left(w_{\varepsilon}\right)\right) \cdot \mathcal{T}_{\varepsilon}\left(\operatorname{rot}\left(\psi_{\varepsilon}\right)\right) d x d y+\varepsilon^{2} R_{c} \int_{\mathbb{R}^{3} \times Y^{*}} \mathcal{T}_{\varepsilon}\left(\operatorname{div}\left(w_{\varepsilon}\right)\right) \cdot \mathcal{T}_{\varepsilon}\left(\operatorname{div}\left(\psi_{\varepsilon}\right)\right) d x d y \\
& \quad+4 N^{2} \int_{\mathbb{R}^{3} \times Y^{*}} \mathcal{T}_{\varepsilon}\left(w_{\varepsilon}\right) \cdot \mathcal{T}_{\varepsilon}\left(\psi_{\varepsilon}\right) d x d y-2 N^{2} \int_{\mathbb{R}^{3} \times Y^{*}} \mathcal{T}_{\varepsilon}\left(\operatorname{rot}\left(\psi_{\varepsilon}\right)\right) \cdot \mathcal{T}_{\varepsilon}\left(u_{\varepsilon}\right) d x d y \\
& \quad+2 N^{2}(\beta-1) \varepsilon^{-1} \int_{\mathbb{R}^{3} \times \partial F}\left(\mathcal{T}_{\varepsilon}^{b}\left(u_{\varepsilon}\right) \times n\right) \cdot \mathcal{T}_{\varepsilon}\left(\psi_{\varepsilon}\right) d x d \sigma(y)=\int_{\mathbb{R}^{3} \times Y^{*}} \mathcal{T}_{\varepsilon}(g) \cdot \mathcal{T}_{\varepsilon}\left(\psi_{\varepsilon}\right) d x d y .
\end{aligned}
$$

Next, we pass to the limit in every terms of the previous variational formulation:

- First to third terms. From convergences (4.64), (4.66) and (4.86), we have

$$
\begin{aligned}
& \varepsilon^{2} \int_{\mathbb{R}^{3} \times Y^{*}} \mathcal{T}_{\varepsilon}\left(\operatorname{rot}\left(w_{\varepsilon}\right)\right) \cdot \mathcal{T}_{\varepsilon}\left(\operatorname{rot}\left(\psi_{\varepsilon}\right)\right) d x d y=\int_{\mathbb{R}^{3} \times Y^{*}} \varepsilon \mathcal{T}_{\varepsilon}\left(\operatorname{rot}\left(w_{\varepsilon}\right)\right) \cdot \varepsilon \mathcal{T}_{\varepsilon}\left(\operatorname{rot}\left(\psi_{\varepsilon}\right)\right) d x d y \\
& \rightarrow \int_{\Omega \times Y^{*}} \operatorname{rot}_{y}(\hat{w}(x, y)) \cdot\left(\eta(x) \operatorname{rot}_{y}(\Psi(y))\right) d x d y=\int_{\Omega \times Y^{*}} \operatorname{rot}_{y}(\hat{w}(x, y)) \cdot \operatorname{rot}_{y}(\eta(x) \Psi(y)) d x d y, \\
& \varepsilon^{2} \int_{\mathbb{R}^{3} \times Y^{*}} \mathcal{T}_{\varepsilon}\left(\operatorname{div}\left(w_{\varepsilon}\right)\right) \cdot \mathcal{T}_{\varepsilon}\left(\operatorname{div}\left(\psi_{\varepsilon}\right)\right) d x d y=\int_{\mathbb{R}^{3} \times Y^{*}} \varepsilon \mathcal{T}_{\varepsilon}\left(\operatorname{div}\left(w_{\varepsilon}\right)\right) \cdot \varepsilon \mathcal{T}_{\varepsilon}\left(\operatorname{div}\left(\psi_{\varepsilon}\right)\right) d x d y \\
& \rightarrow \int_{\Omega \times Y^{*}} \operatorname{div}_{y}(\hat{w}(x, y)) \cdot\left(\eta(x) \operatorname{div}_{y}(\Psi(y))\right) d x d y=\int_{\Omega \times Y^{*}} \operatorname{div}_{y}(\hat{w}(x, y)) \cdot \operatorname{div}_{y}(\eta(x) \Psi(x, y)) d x d y . \\
& \int_{\mathbb{R}^{3} \times Y^{*}} \mathcal{T}_{\varepsilon}\left(w_{\varepsilon}\right) \cdot \mathcal{T}_{\varepsilon}\left(\psi_{\varepsilon}\right) d x d y \rightarrow \int_{\Omega \times Y^{*}} \hat{w}(x, y) \cdot(\eta(x) \Psi(y)) d x d y .
\end{aligned}
$$

- Fourth term. From (4.55) and (4.86), we get

$$
\begin{aligned}
\int_{\mathbb{R}^{3} \times Y^{*}} \mathcal{T}_{\varepsilon}\left(\operatorname{rot}\left(\psi_{\varepsilon}\right)\right) \cdot \mathcal{T}_{\varepsilon}\left(u_{\varepsilon}\right) d x d y & =\int_{\mathbb{R}^{3} \times Y^{*}} \varepsilon \mathcal{T}_{\varepsilon}\left(\operatorname{rot}\left(\psi_{\varepsilon}\right)\right) \cdot \varepsilon^{-1} \mathcal{T}_{\varepsilon}\left(u_{\varepsilon}\right) d x d y \\
& \rightarrow \int_{\Omega \times Y^{*}} \eta(x) \operatorname{rot}_{y}(\Psi(y)) \cdot \hat{u}(x, y) d x d y=\int_{\Omega \times Y^{*}} \operatorname{rot}_{y}(\eta(x) \Psi(y)) \cdot \hat{u}(x, y) d x d y .
\end{aligned}
$$

- Fifth term. From convergence (4.56) and (4.86),

$$
\left.\varepsilon^{-1} \int_{\mathbb{R}^{3} \times \partial F}\left(\mathcal{T}_{\varepsilon}^{b}\left(u_{\varepsilon}\right) \times n(y)\right)\right) \cdot \mathcal{T}_{\varepsilon}\left(\psi_{\varepsilon}\right) d x d \sigma(y) \rightarrow \int_{\Omega \times \partial F}(\hat{u}(x, y) \times n(y)) \cdot(\eta(x) \Psi(y)) d x d \sigma(y) .
$$

- Sixth term. From Proposition 4.127 and convergence (4.86),

$$
\int_{\mathbb{R}^{3} \times Y^{*}} \mathcal{T}_{\varepsilon}(g) \cdot \mathcal{T}_{\varepsilon}\left(\psi_{\varepsilon}\right) d x d y \rightarrow \int_{\mathbb{R}^{3} \times Y^{*}} g(x) \cdot(\eta(x) \Psi(y)) d x d y .
$$

From the previous convergences and noting $\psi(x, y)=\eta(x) \Psi(y)$, we obtain

$$
\begin{aligned}
& R_{c} \int_{\Omega \times Y^{*}} \operatorname{rot}_{y}(\hat{w}(x, y)) \cdot \operatorname{rot}_{y}(\psi(x, y)) d x d y+R_{c} \int_{\Omega \times Y^{*}} \operatorname{div}_{y}(\hat{w}(x, y)) \cdot \operatorname{div}_{y}(\psi(x, y)) d x d y \\
& \quad+4 N^{2} \int_{\Omega \times Y^{*}} \hat{w}(x, y) \cdot \psi(x, y) d x d y-2 N^{2} \int_{\Omega \times Y^{*}} \operatorname{rot}_{y}(\psi(x, y)) \cdot \hat{u}(x, y) d x d y \\
& \quad+2 N^{2}(\beta-1) \int_{\Omega \times \partial F}(\hat{u}(x, y) \times n(y)) \cdot \psi(x, y) d x d \sigma(y)=\int_{\Omega \times Y^{*}} g(x) \cdot \psi(x, y) d x d y,
\end{aligned}
$$


which, by density, holds for $\hat{\psi} \in \mathcal{V}$.

Finally, under condition (3.26), we can prove that the variational formulation given by (4.84) and (4.87) admits a unique solution $(\hat{u}, \hat{w})$ in $\mathcal{V}^{0}\left(Y^{*}\right) \times \mathcal{V}$ (see proof of Lemma 4.17 above), and so we conclude that the whole sequence $\left(\varepsilon^{-1} \mathcal{T}_{\varepsilon}\left(u_{\varepsilon}\right), \mathcal{T}_{\varepsilon}\left(w_{\varepsilon}\right)\right)$ converges to this solution. Reasoning as in [1], it follows that there exists $q(x) \in L^{2}\left(Y^{*}\right) / \mathbb{R}$ and $\hat{q}(x, y) \in L^{2}\left(\Omega ; L_{\text {per }}^{2}\left(Y^{*}\right) / \mathbb{R}\right)$ such that the variational formulation given by (4.84) and (4.87) is equivalent to system (4.73)-(4.77). It remains to prove that $q$ coincides with pressure $p$. This can be easily done by proceeding as above by considering a test function which is divergence-free only in $y$, and identifying limits.

Step 2. In this step, we eliminate the microscopic variable $y$ in the homogenized problem (4.73)-(4.81) and then, we obtain a Darcy equation for the pressure $p$. To do that, for every $k=1,2, i=1,2,3$, we consider the micropolar local problem (3.29), which has a unique solution $\left(u^{i, k}, w^{i, k}, \pi^{i, k}\right) \in V_{Y}^{0} \times V_{Y} \times L_{0}^{2}\left(Y^{*}\right)$ (see Lemma 4.17 below). Then, by the following indentification

$$
\begin{aligned}
& \hat{u}(x, y)=\sum_{i=1}^{3}\left(\left(f_{i}(x)-\partial_{x_{i}} p(x)\right) u^{i, 1}(y)+g_{i}(x) u^{i, 2}(y)\right), \\
& \hat{w}(x, y)=\sum_{i=1}^{3}\left(\left(f_{i}(x)-\partial_{x_{i}} p(x)\right) w^{i, 1}(y)+g_{i}(x) w^{i, 2}(y)\right), \\
& \hat{q}(x, y)=\sum_{i=1}^{3}\left(\left(f_{i}(x)-\partial_{x_{i}} p(x)\right) \pi^{i, 1}(y)+g_{i}(x) \pi^{i, 2}(y)\right),
\end{aligned}
$$

and thanks to identities (4.58) and (4.67), we deduce that $u$ and $w$ have the expressions given in (3.27).

Next, the divergence condition with respect to the variable $x$ given in (4.61) together with the expression of $u$ gives (3.28).

Finally, positive definiteness of $K^{(1)}$ follows from the fact that $K_{i j}^{(1)}=\mathcal{A}_{Y}\left(u^{i, 1}, w^{i, 1} ; u^{j, 1}, w^{j, 1}\right)$ and the coercivity of the bilinear form $\mathcal{A}_{Y}$ defined by (4.72). Since (3.28) is an elliptic equation with $K^{(1)} f+K^{(2)} g \in L^{2}(\Omega)$, then it has a unique solution $p \in H^{1}(\Omega) \cap L_{0}^{2}(\Omega)$, and $u, w \in L^{2}(\Omega)^{3}$ given by (3.27) are also unique. By the uniqueness of the limits, the whole sequences converge.

\section{References}

[1] G. Allaire, Homogenization of the Stokes flow in a connected porous medium. Asymp. Anal., 2 (1989) 203-222.

[2] G. Allaire, Homogenization of Navier-Stokes equations with a slip boundary condition. Commun. Pure Appl. Math., 44 (1991) 605-641.

[3] I. Aganovic, Z. Tutek, Nonstationary micropolar fluid flow through porous medium, Nonl. Anal. Teo. Meth. Appl., 30 (1997) 3171-3178.

[4] C. Amrouche, C. Bernardi, M. Dauge, V. Girault, Vector potentials in three-dimensional nonsmooth domains, Math. Meth. Appl. Sci., 21(1998) 823-864.

[5] M. Anguiano, Existence, uniqueness and homogenization of nonlinear parabolic problems with dynamical boundary conditions in perforated media, Mediterr. J. Math., (2020) 17:18.

[6] G. Bayada, N. Benhaboucha, M. Chambat, New models in micropolar fluid and their applications to lubrication, Math. Mod. Meth. Appl. S., 15 (2005) 343-374.

[7] G. Bayada, N. Benhaboucha, M. Chambat, Wall slip induced by a micropolar fluid, J. Eng. Math., 60 (2008) $89-100$. 
[8] G. Bayada, M. Chambat, S.R. Gamouana, About thin film micropolar asymptotic equations, Quart. Appl. Math., 59 (2001) 413-439.

[9] G. Bayada, M. Chambat, S.R. Gamouana, Micropolar effects in the coupling of a thin film past a porous medium, Asymp. Anal., 30 (2002) 187-216.

[10] G. Bayada, G. Lukaszewicz, On micropolar fluids in the theory of lubrication. Rigorous derivation of an analogue of the Reynolds equation, Internat. J. Eng. Sci., 34 (1996) 1477-1490.

[11] N.M. Bessonov, Boundary viscosity conception in hydrodynamical theory of lubrication. Russian Academy of Sicence, Inst. Prob. Mech. Eng. St-Petersbourg, 81 (1993) 105-108.

[12] N.M. Bessonov, A new generalization of the Reynolds equation for a micropolar fluid and its application to bearing theory, Tribol. Int., 27 (1994) 105-108.

[13] F. Boyer, P. Fabrie, Mathematical tools for the study of the incompressible Navier-Stokes equations and related models. Springer, New York, 2012.

[14] A. Capatina, H. Hene, Homogenisation of the Stokes problem with a pure non-homogeneous slip boundary condition by the periodic unfolding method. Euro. J. App. Math., 22 (2011) 333-345.

[15] D. Cioranescu, A. Damlamian, G. Griso, Periodic unfolding and homogenization, C.R. Acad. Sci. Paris, Ser. I, 335 (2002) 99-104.

[16] D. Cioranescu, P. Donato, H. Ene, Homogenization of the Stokes Problem With Non-homogeneous Slip Boundary Conditions, Math. Meth. Appl. Sci., 19 (1996) 857-881.

[17] D. Cioranescu, P. Donato, R. Zaki, The periodic unfolding method in perforated domains, Port. Math., 63 (2006) 467-496.

[18] D. Cioranescu, P. Donato, R. Zaki, Asymptotic behaviour of elliptic problems in perforated domains with nonlinear conditions. Asymptotic Anal., 53 (2007) 209-235.

[19] D. Cioranescu, A. Damlamian, G. Griso, The periodic unfolding method. Theory and Applications to Partial Differential Problems. Series in Contemporary Mathematics 3, 2018.

[20] D. Cioranescu and J. Saint Jean Paulin, Homogenization in open sets with holes, J. Math. Anal. Appl., 71 (1979) 590-607.

[21] C. Conca, On the application of the homogenization theory to a class of problems arising in fluid mechanics. J. Math. Pures Appl., 64 (1985) 31-75.

[22] A. Damlamian, An elementary introduction to periodic unfolding. Gakuto Int. Ser., Math. Sci. Appl., 24 (2006) 119-136.

[23] P. G. Darcy, Les fontaines publiques de la ville de Dijon Paris. Victor Dalmont, 1856.

[24] G. Duvaut, J.L. Lions, Les inequations en Mécanique et en Physique, Dunod, Paris, 1972.

[25] A. C. Eringen, Theory for micropolar fluid, J. Math. Mech., 16 (1966) 1-16.

[26] G.P. Galdi, An introduction to the mathematical theory of the Navier-Stokes equations, Springer-Verlag, 1994.

[27] V. Girault, P. A. Raviart, Finite Element Approximation of the Navier Stokes Equation, Springer-Verlag (1979).

[28] P. Grisvard, Elliptic Problems in Nonsmooth Domains. Pitman, Boston, 1985.

[29] G. Lukaszewicz, Micropolar fluids, theory and applications, Modeling and Simulation in Science, Engineering and Technology, Birkhaüser, 1999. 
[30] N.P. Migun, On hydrodynamic boundary conditions for microstructural fluids, Rheol. Acta, 23 (1984) 575-581.

[31] P.P. Prokhorenko, N.P. Migun, S.V. Grebenshchicov, Experimental studies of polar indicator liquids used in capillary penetrant testing, Internat. J. Eng. Sci., 25 (1947) 482-489.

[32] L. Tartar, Incompressible fluid flow in a porous medium convergence of the homogenization process. Appendix to Lecture Notes in Physics, 127 (1980).

[33] R. Temam, Navier-Stokes Equations, North Holland, 1984.

[34] M. Vanninathan, Homogenization of eigenvalues problems in perforated domains, Proc. Indian Acad. Sci., 90 (1981) 239-271.

[35] R. Zaki, Homogenization of a Stokes problem in a porous medium by the periodic unfolding method, Asymp. Anal., 79 (2012) 229-250. 